\title{
A VARIATIONAL-AUTOENCODER APPROACH TO SOLVE THE HIDDEN PROFILE TASK IN HYBRID HUMAN-MACHINE TEAMS
}

\author{
A PREPRINT
}

\author{
Niccoló Pescetelli * \\ New Jersey Institute of Technology \\ 323 Dr Martin Luther King Jr Blvd, Newark, NJ 07102 \\ USA \\ niccolo.pescetelli@njit.edu
}

Patrik Reichert

School of Business, Economics and Law at the University of Gothenburg

Gothenburg, 40530

Sweden

patrik.reichert@economics.gu.se

Alex Rutherford

Max Planck Institute for Human Development

Berlin, 14195

Germany

rutherford@mpib-berlin.mpg.de

November 18, 2021

\begin{abstract}
Algorithmic agents, popularly known as bots, have been accused of spreading misinformation online and supporting fringe views. Collectives are vulnerable to hidden-profile environments, where taskrelevant information is unevenly distributed across individuals. To do well in this task, information aggregation must equally weight minority and majority views against simple but inefficient majoritybased decisions. In an experimental design, human volunteers working in teams of 10 were asked to solve a hidden-profile prediction task. We trained a variational auto-encoder (VAE) to learn people's hidden information distribution by observing how people's judgements correlated over time. A bot was designed to sample responses from the VAE latent embedding to selectively support opinions proportionally to their under-representation in the team. We show that the presence of a single bot (representing 10\% of team members) can significantly increase the polarization between minority and majority opinions by making minority opinions less prone to social influence. Although the effects on hybrid team performance were small, the bot presence significantly influenced opinion dynamics and individual accuracy. These findings show that self-supervised machine learning techniques can be used to design algorithms that can sway opinion dynamics and group outcomes.
\end{abstract}

Keywords Social bot $\cdot$ decision-making $\cdot$ hybrid intelligence $\cdot$ hidden profile $\cdot$ team performance

\section{Introduction}

The effect and influence of minority opinions on social groups have long been studied in psychology and sociology [1, 2, 3, 4]. More recently, public interest in these questions has increased due to media coverage of troll factories,

${ }^{*}$ Corresponding author. 
social bots and automated accounts on public fora [5, 6]. However, few studies have experimentally measured social bots' ability to selectively support minority opinions and influence collective outcomes. In this paper, we trained a variational auto-encoder (VAE) to recognize shared information sources among a team of people solving a hidden profile task. We then used the VAE model to program a bot to selectively support minority opinions and quantified the effect on teams opinions and teams accuracy.

The presence of automated accounts, popularly known as social bots, is well documented on social media [7, 8]. Their goals and tasks can vary from innocuous chatbots to more malicious automated accounts [9]. Social media bots, in particular, have been accused of spreading hyper-partisan content to push political agendas and increase the perception of fringe views[10]. Bots may inflate the perception of particular views' popularity and contribute to the spread of misinformation, conspiratory theories, and partisan messages [11, 5]. Whether bots can be intentionally designed to improve group performance is a matter of debate [12, 13]

Collectives, like groups, teams and online crowds, are vulnerable when information to solve a task is unequally distributed across multiple players [14, 15]. This scenario is known in psychology as the hidden profile problem. Some pieces of information are shared by a majority of players, while other pieces of information are known only by a minority of players (hidden information). The typical finding is that players tend to discuss and use information shared by the majority rather than the (still useful) information available only to minority players. We hypothesized that, in this scenario, artificially supporting minority opinions should improve team performance by rebalancing information distribution in the group. The hidden profile paradigm offers the opportunity to test the effect of a bot supporting minority views in a task where such influence can be beneficial for group outcomes.

Threats (and opportunities) of deploying bots in social networks are more consequential when people's opinions are aggregated to form collective decisions like during democratic processes and elections, or when opinions are aggregated to forecast future outcomes in economic and geopolitical forecasting. For instance, researchers have documented bots' potential for distorting information and exerting undue influence on popular opinions in real-world democratic decisions [5, 16, 17, 18]. These concerns have stimulated research on the subject [19, 20, 21, 22] and have mobilized platforms to improve automatic detection and removal of algorithmic accounts [23, 24, 25, 16]. We contribute to this literature by advancing the hypothesis that, in addition to removing malicious bots, an equally effective strategy to combat misinformation is to design bots expressing views that increase opportunities for individuals to be exposed to hidden information.

In this paper, we experimentally study these phenomena in a modified hidden profile paradigm [14, 15]. We asked teams of volunteers to make weather predictions based on random indicators (humidity, temperature and wind speed) with individual predictive patterns, which had to be learned from trial and error. Although outcomes were predicted by a linear combination of all indicator variables, each individual was able to observe only one indicator. Indicators were unequally distributed among the team, with one indicator being shown to the majority of participants (majority group, $50 \%$ ), while two more being shown to two minority groups (30\% and $20 \%$ respectively). In each round, participants were asked to give an independent forecast and then revise it based on the forecasts made by others in their team.

We hypothesize that a bot selectively supporting minority views would help team performance by reducing the majority's influence in this task. Instead of telling the bot whether people held majority or minority information sources, we trained a variational autoencoder (VAE) to learn this representation in an unsupervised fashion, namely from observing the correlation of people's responses over the first 110 rounds of the game. VAE-based approaches are used in several domains to generate low-dimensional interpretable embeddings from complex data [26]. The use of a VAE allowed us to generate original opinions by sampling from the hidden latent representation layer of the VAE, rather than simply copying of human opinions [25, 27]. Our bot used VAE's representation of people's similarity (based on past trials) to strategically position itself on the opinion space. In the last 50 rounds of the game, we replaced a human player in the review stage with the VAE bot. Bot's responses were inbetween the average majority response and the minority response, but closer to minority views. Thus the bot positioned itself in a region of opinions where people are more susceptible to social influence, sometimes referred to as 'latitude of acceptance' [4]. This strategy may be more flexible and adaptive than simple opinion copying because it is adaptive to opinion shifts in humans over time. While current bots may have a significant impact because of their sheer number, strategic adaptive bots may be individually more effective at influencing the social system they are plugged in.

Furthermore, we hypothesized that by adopting opinions between majority and minority views, the bot could bridge the gap between the two opinion clusters and facilitate consensus. We compare the effect of such an adaptive bot with a random bot [28]. We conducted a series of exploratory data analysis that we report below. Our findings suggest that instead of bridging the gap between majority and minority views, the VAE bot further increased the team's polarisation by making minority views less prone to social influence by the majority. Reduced majority influence was especially true when people were facing challenging rounds, and thus people were more likely to seek social information. The 


\section{a Training phase}

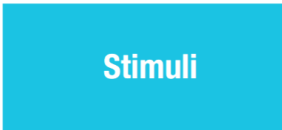

b Interaction phase
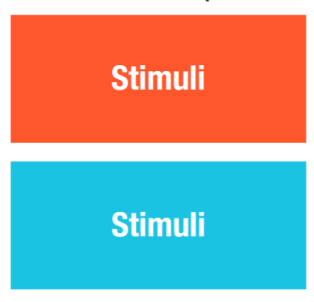

Stimuli probability.

bot's influence on team performance was relatively weak but followed a similar pattern. In minority groups, the VAE

\section{Methods}

Procedure The game was implemented in Empirica [29]. The game's task consisted in predicting a binary weather outcome (Rain $v s$. No Rain) based on the information provided by independent weather indicators. Participants were in teams of ten. Within each team, participants were assigned to one of three weather conditions, either "wind",

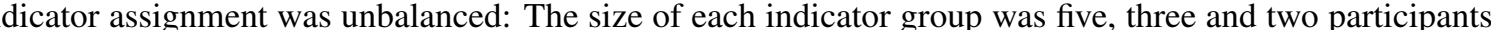
run.

The training phase had 110 rounds. In each round, a participant could see a measurement ("stimuli") on a percentage scale after which she was asked to predict the chances of rain within a 10 second window. The prediction was provided on a probabilistic scale of 0 to 1 , where 1 corresponded to being certain that it will rain and 0 corresponded to being certain that it will not rain. After each round, participants could see the outcome for 5 seconds, and the Brier score culated from their predictions was added to their score total. We used Brier scores to discourage over- and/or under-confidence. Brier scores were computed as:

$$
b=(I-p)^{2}+(\neg I-\neg p)^{2}
$$

Where $\mathrm{I}$ is the indicator variable (I=0: sun; I=1: rain), p is the probabilistic forecast and " $\neg$ " represents the complementary

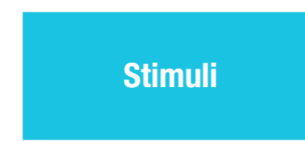

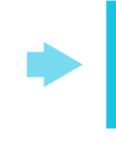
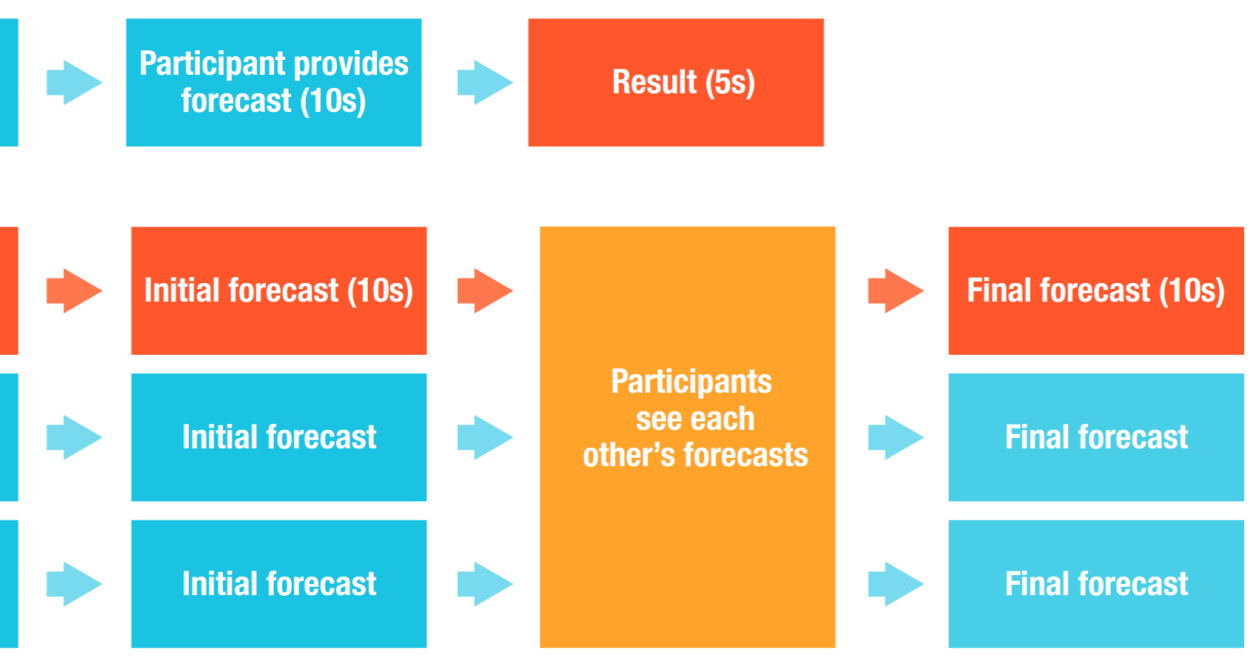

Figure 1: Experiment Timeline. (a) During the first 110 rounds, participants see measurements from their indicator group and need to provide weather forecasts. On each round they receive feedback ("It rained" vs. "It did not rain"), thus allowing them to learn the predictive relation between the indicator and the outcome. (b) During the interaction phase, after seeing their indicator measurements and providing their independent answer, participants could see the forecasts made by other participants, before receiving round feedback.

In the interaction phase lasting for 50 rounds, participants continued to see measurements for their respective indicator category (wind, temperature, humidity), but their forecast was now shared with other participants in their team. After seeing each other's forecasts, participants had 10 seconds to revise their initial guess and provide a final forecast for every round. 
During the interaction phase, we included an algorithmic player in each team. In 15 of the 30 teams (treatment teams) this algorithmic player provided forecasts based on the VAE model we trained on the respective team's training phase data (Figure 1). In the other 15 teams (control teams), the algorithmic player provided randomly generated guesses. We compared the effect of including the VAE algorithm as an algorithmic player in a team on performance compared to a random baseline.

Stimuli The stimuli $x_{(\cdot)}$ for temperature, humidity and wind were randomly generated for all rounds of the game prior to its start. The values generated were then transformed into predictor values. The function used for this transformation was different for each predictor:

$$
\begin{gathered}
\text { predictor value } \text { hum }^{\text {hum }}=1+x_{\text {hum }}+0.01 \epsilon \\
\text { predictor value }{ }^{\text {temp }}=1-x_{\text {temp }}+0.01 \epsilon \\
\text { predictor value }^{\text {wind }}= \begin{cases}2, & x_{\text {wind }} \geq 2 \\
50 x_{\text {wind }}^{2}-50 x_{\text {wind }}+10+0.01 \epsilon, & -2<x_{\text {wind }}<2, \\
-2, & x_{\text {wind }} \leq-2\end{cases}
\end{gathered}
$$

Where $\epsilon \sim N(0,1)$ is a random noise term. These functions (ignoring the random error component) led to a positive linear relationship in the case of humidity, a negative linear relationship for temperature, and a semi-quadratic relationship for wind (see Figure S1). In other words, chances of rain were highest for (1) high humidity values; (2) low temperature values; and (3) low and high wind values.

Both stimuli and the predictor values for all 160 rounds were generated before the game started for a given team. Taking these 160 values for each predictor category as the "sample", we transformed the predictor values to $\mathrm{z}$-scores. Negative z-scores had a negative effect on rain probability, while positive z-scores increase the likelihood of rain.

We then summed up the three z-scores - one for wind, temperature, and humidity each - from a given round, and transformed the resulting sum using a sigmoid function to get our rain probability for round $k$ (see Figure S2):

$$
P\left(\operatorname{rain}_{k}=1 \mid Z_{k}\right)=\frac{1}{\left.1+\exp \left(-5 Z_{k}\right)\right)}
$$

Where $Z_{k} \equiv z_{k}^{\text {temp }}+z_{k}^{\text {hum }}+z_{k}^{\text {wind }}$ for round $k$.

Variational autoencoder We used our two-stage experiment to train a variational autoencoder model ("VAE" in the following) on data collected directly from participants during the first stage of the experiment ("Training phase"), and use it as an algorithmic player in the second part of the experiment ("Interaction phase"). We compared behavior in groups where the algorithmic player followed the VAE with groups where the algorithmic player provided random responses.

The VAE model was implemented using the Keras.js package and trained it on the first 110 rounds (Training phase) with a burn-in of 10 rounds to allow the players to familiarize with the task (Figure 2). An autoencoder is a type of convolutional neural network that converts a high-dimensional input into a low-dimensional one (the latent vector), and tries to reconstruct the original input with the highest quality possible from this vector. Our VAE consisted of two connected networks, one encoder and one decoder. When training the model, the encoder took as input the response matrix $X$ collected during the training phase. Each participant in the game was treated as one feature and responses on each round were treated as different observations. The encoder returned a vector of lower dimensionality compared to the initial input features. The low dimensional space was a hidden layer with three nodes. This procedure compresses redundant information arising from response correlations among participants, akin to other dimensionality reduction techniques such as principal component analysis (PCA). The VAE differs in that (i) the encoding and decoding process is in general non-linear and (ii) the hidden nodes learn a distribution over latent variables and so their response is probabilistic. The architecture of the VAE requires many hyper-parameters to be set and optimised typically. In this study our aim is to prove that a VAE can perform the function of a minority supporting bot, rather than to optimise this behaviour. The latent vector was then fed to the decoder that was trained to reconstruct the original data by minimizing the difference between the input matrix $X$ and the output matrix $X^{\prime}$. The advantage of this technique compared to other dimensionality reduction techniques such as principal component analysis is that the latent space can be used to generate new data that has never seen by the model. In our weather forecasting task, this results in a bot that is able to 


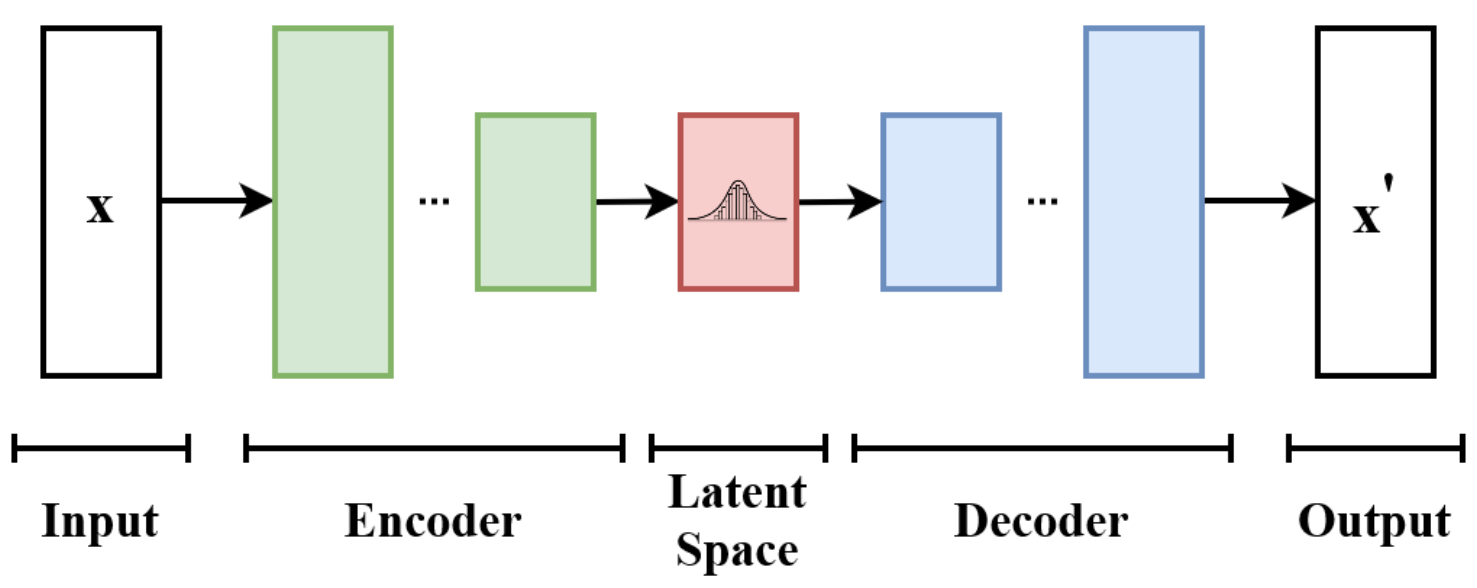

Figure 2: Schematic architecture of a variational auto-encoder. The input X is compressed by the encoder layer (green) into a hidden representational layer (red). A decoder (blue) takes as input the compressed representation and attempts to reproduce the original input as closely as possible (X'). Credit:EugenioTL, Wikipedia Commons contributor (CC BY-SA 4.0)

produce forecasts that were not made by any other human player. To train the model we used defaults parameters in the Keras.js package.

After training, we proceeded in three steps to to create an algorithmic agent that could operate in the second stage ("Interaction phase"): (1) we apply K-means $(\mathrm{k}=3)$ to the last output layer's weights; (2) we create a new set of coordinates along the hidden layer (corresponding to a new set of weights) by computing the weighted average of cluster centroids obtained in 1; (3) we replace the weights of the last output layer of the VAE with this new set of weights.

1. We tested this procedure in a computer simulation using synthetic data prior to running the experiment. The code is publicly available on Open Science Framework together with the rest of the analyses accompanying this paper. We show that the last layer's weights correctly separate participants by condition. In other words, the participant distance along the hidden layer represents, after training, their similarity of responses across rounds. Figure 3 reproduce the same weights analysis with one of the teams tested. Figure 3 a shows the correlation coefficients of the 10 sets of weights in the last layer of the model. High correlation coefficients represent greater weight similarity. The model learned to assign similar weights to participants giving similar responses. Similarly, figure $3 \mathrm{~b}$ shows a 2-dimensional projection of the output weights. Each blue point corresponds to one participant in a test team. A lower distance in the projected space corresponds to a similar pattern of weights from the hidden layer to the output layer of the VAE.

2. The algorithmic participant's profile was specific for each team of participants and was defined as a new set of three coordinates along the hidden layer. The set of coordinates was obtained by averaging the clusters centroids, using weights $\mathbf{w}$ inversely proportional to the number of participants $N$ in each cluster $c$ :

$$
w_{c}=\left(1 / N_{c}\right)^{\gamma}
$$

where $\gamma$ is the parameter controlling the bias towards supporting minority views (here set to 2). Figure 3p shows a 2-d projection of the set of weight that this procedure assigned to the algorithmic participant in one of the teams recruited (orange dot). Notice that the bot has a shorter distance (i.e.more similar weights) from the two smaller clusters (minority opinion clusters) than to the larger cluster (majority opinion cluster).

3. After defining the algorithmic player's profile we used it to sample a new response on each subsequent round (Interaction phase). To do that, we replaced at random one output unit of the VAE (corresponding to one human player) with our new set of weights representing the algorithmic player. We then run this new VAE model on all subsequent new (human) forecasts to obtain the responses of our algorithmic player. The net effect is that the VAE bot produces an original (unseen) forecast based on the pattern of forecasts produced by other human players. 

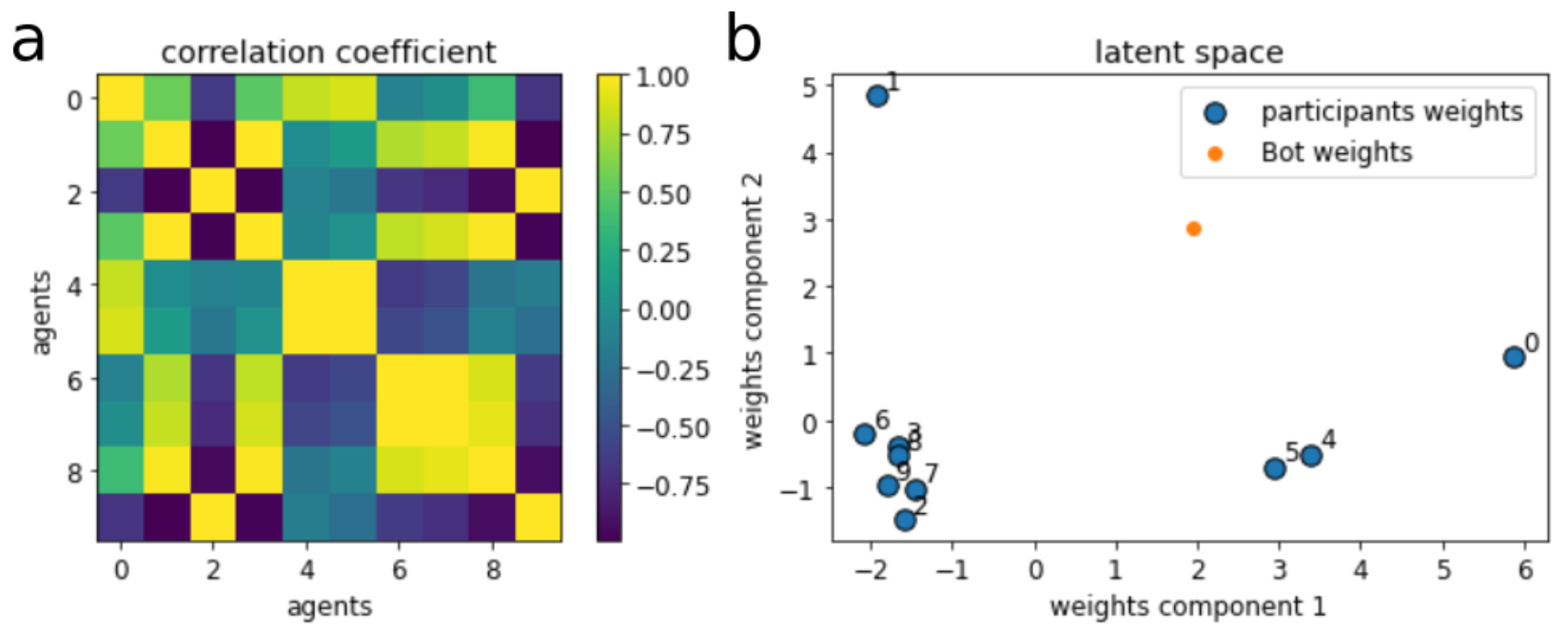

Figure 3: Example of a latent space of a variational autoencoder model trained on one of the teams in the study. (a) Correlation coefficient of the weights to the output layer of the variational autoecoder, after training. Clear structure of the data, as learned by the model, is seen in the clusters of high correlation coefficients. (b) The weights to the output layer projected on a 2-dimensional surface using principal component analysis. Three distinct clusters can be seen in the figure, each corresponding to a team of participants with similar response patterns. The model learned distinct representations corresponding to one large cluster (majority opinion) and two smaller clusters (minority opinions) as shown by the clear separation of the model's weights in latent space. The bot player is represented in orange and shows a response pattern that is closer to the smaller clusters (minority opinions) than to the larger cluster (majority opinions).

Participants The experiment was ran double-blind on Prolific.co. We allocated incoming participants randomly between the control and treatment team, until we reached the desired 30 teams ( 15 control and 15 treatment).

We paid participants $£ 8$ to complete the experiment that was estimated to take 55 minutes (equivalent to $£ 8.57 /$ hour). On top of the flat compensation rate, participants were ranked within their group of 10 based on their score achieved during the experiment. The participant with the highest score received an additional $£ 10$ and the participant ranked second received a bonus of $£ 5$. We discuss the potential downsides of tournament incentives later in the paper.

Our participant pool is diverse compared to most laboratory experiments using student subject pools, helping us to ease some of the concerns regarding the external validity of our findings. The summary statistics of our sample demographic information is presented in the Supplementary Table S1. The mean and median age of participants is 31.8 years and 30 years, respectively (with a standard deviation of approximately 10 years). The sample is balanced with respect to gender, having $52.4 \%$ male participants.

Most participants (64.5\%) reported English as their first language and the most represented nationalities with respect to the total number of participants were the UK (38.8\%) and the US (21.1\%). $47.8 \%$ of participants were full-time employed and students represented $30.8 \%$ of our sample.

\section{Results}

Team polarization We first tested whether the VAE bot helped reduce team polarization. Although the VAE player supported minority views more than majority views, it also represented a (weighted) average of each opinion cluster. Thus, we expected the VAE bot to bridge the gap between opinion clusters and increase alignment [30]. To test this hypothesis, we compared polarization in teams assigned to the VAE condition with polarization in control teams. We measured polarization as the standard deviation of forecasts [31], which we calculated for forecast distributions both before (pre SD) and after (post SD) exposure to peer information. Our measure of interest was $\Delta \mathrm{SD}$, defined as the change in the standard deviation of forecasts before and after peer exposure.

Participants saw different information according to which predictor group they were assigned to. Therefore, if one predictor suggested a high likelihood of rain while another the opposite, this would itself result in a higher standard 
deviation of forecasts. To control for these spurious effects, we measure $Z$ Range, which calculates the distance between the largest and smallest $\mathrm{z}$-score of the three predictor values in each round:

$$
\text { ZRange }_{j, k}=\max \left(z_{j, k}^{\text {temp }}, z_{j, k}^{\text {hum }}, z_{j, k}^{\text {wind }}\right)-\min \left(z_{j, k}^{\text {temp }}, z_{j, k}^{\text {hum }}, z_{j, k}^{\text {wind }}\right)
$$

For team $j$ and round $k$. We ran two mixed effects model with random effect for teams (nested within the date the game was ran on). The first model tested whether the change in polarization $\Delta \mathrm{SD}$ was affected by our bot treatment (dummy coding: 1 = VAE bot, 0 = random bot), and controlling for $\mathrm{z}$-score range. In the second model, the dependent variables was changed to post-interaction polarization (post SD). Results are shown in Table 1

Post-interaction polarization also tended to be higher in VAE condition compared to the control $(\beta=.35, p=.052)$. As expected, a higher $Z$ Range predicted increased polarization, but did not affect the change in polarization $\Delta \mathrm{SD}$. We thus focused on $\triangle \mathrm{SD}$ as this was less affected by round-by-round fluctuations in the indicators' range. The presence of VAE bots significantly increased polarization, compared to a random bot baseline $(\beta=.28, p=.01)$. Overall these findings suggest that although the bot provided responses that were in between minority and majority opinions, its bias to support minority opinions more than majority opinions led to an overall increase in polarization compared to the baseline.

\begin{tabular}{|c|c|c|c|c|}
\hline Dependent Variable: & \multicolumn{2}{|c|}{ post SD } & \multicolumn{2}{|c|}{$\Delta \mathrm{SD}$} \\
\hline Predictors & Estimate & $p$-value & Estimate & $p$-value \\
\hline (Intercept) & -0.18 & 0.169 & -0.14 & 0.086 \\
\hline Treatment & 0.35 & 0.052 & $0.28 *$ & 0.012 \\
\hline Z Range & $0.11^{* * *}$ & $<0.001$ & -0.03 & 0.416 \\
\hline Random effects & \multicolumn{2}{|c|}{ Yes } & \multicolumn{2}{|c|}{ Yes } \\
\hline Observations & \multicolumn{2}{|c|}{1500} & \multicolumn{2}{|c|}{776} \\
\hline Marginal / Conditional $\mathrm{R}^{2}$ & \multicolumn{2}{|c|}{$0.043 / 0.270$} & \multicolumn{2}{|c|}{$0.021 / 0.073$} \\
\hline
\end{tabular}

Table 1: Mixed Models for Team Polarization. The presence of the VAE bot in hybrid teams increased the likelihood of polarization and the spread of forecasts post social interaction.

Influence of minority opinion One hypothesis for the increased polarization observed in VAE condition was that the VAE bot, by supporting minority views, increased the influence of people holding those views against a majority. The presence of the VAE bot may have made people holding minority opinions less prone to social influence. If the minority was more influential, one would expect that final forecasts of majority groups would shift closer to the minority's initial forecasts. We thus looked at whether final forecasts of the two largest sub-groups (the 5 person majority and the 3 person mid-size condition) in teams with the VAE bot were closer to initial minority forecasts. We calculated Minority Influence as the absolute difference between the 2-person minority's median initial forecast and median final forecast of 3 and 5-person sub-groups separately, for each team $j$ and round $k$ :

$$
\begin{gathered}
\operatorname{Minority}_{\text {Influence }_{j, k}^{\text {majority }}}^{\text {mation }}=1-\left|\operatorname{med}\left(\operatorname{prediction}_{I_{j, k}^{\text {minority }}}^{\text {initial }}\right)-\operatorname{med}\left(\operatorname{prediction}_{I_{j, k}^{\text {majority }}}^{\text {final }}\right)\right| \\
\text { Minority Influence }_{j, k}^{\text {mid-size }}=1-\left|\operatorname{med}\left(\operatorname{prediction}_{I_{j, k}^{\text {minority }}}^{\text {initial }}\right)-\operatorname{med}\left(\operatorname{prediction}_{I_{j, k}^{\text {find-size }}}^{\text {final }}\right)\right|
\end{gathered}
$$

Where $I_{j, k}^{\text {minority }}, I_{j, k}^{\text {mid-size }}$ and $I_{j, k}^{\text {majority }}$ denotes participants, who are assigned to the sub-group with a total of two, three and five participants, respectively. The variable is calculated for each team $j$ and for each round $k$. A greater value indicates a greater influence of the minority cluster.

We control for round difficulty for 3 and 5-person sub-groups, as any outside sub-group influence is likely to be diminished in easy rounds (see Supporting Information for a calculation of round difficulty). We calculate Round Difficulty depending on which predictor category (temperature, humidity or wind) was assigned to the majority condition $(\mathrm{N}=5)$ and the mid-size condition $(\mathrm{N}=3)$. This was done to take into account the fact that, for instance, a linear relationship (e.g.temperature) was easier to learn than a quadratic one (e.g.wind).

We tested separately minority influence on the majority sub-group (5-players) and on the mid-size sub-group (3-players) by running mixed effects model with random effect for condition, nested in the date the game was ran. We regressed the treatment dummy ( 1 if bot is VAE, 0 if random), round difficulty and their interaction terms on Minority Influence. 
The results in Table 2 suggests that the VAE bot reduced minority influence on the mid-sized groups in easy rounds $(\beta=-0.36, p=.03)$. However, in teams with the VAE bot, minority influence on the majority increased when the majority cluster faced difficult rounds, and thus people were most uncertain $(\beta=0.31, p<0.001)$.

\begin{tabular}{|c|c|c|c|c|}
\hline \multirow{2}{*}{$\begin{array}{l}\text { Dependent Variable: } \\
\text { Predictors }\end{array}$} & \multicolumn{2}{|c|}{ Minority Influence ${ }^{\text {majority }}$} & \multicolumn{2}{|c|}{ Minority Influence ${ }^{\text {mid-size }}$} \\
\hline & Estimate & $p$-value & Estimates & $p$-value \\
\hline (Intercept) & 0.07 & 0.368 & 0.22 & 0.064 \\
\hline Treatment & -0.14 & 0.181 & $-0.36 *$ & 0.030 \\
\hline Majority Difficulty & $0.13 *$ & 0.014 & & \\
\hline Treatment : Majority Difficulty & $0.31 * * *$ & $<0.001$ & & \\
\hline Mid-Size Difficulty & & & $0.21 * * *$ & $<0.001$ \\
\hline Treatment : Mid-Size Difficulty & & & 0.02 & 0.784 \\
\hline Random effects & \multicolumn{2}{|c|}{ Yes } & \multicolumn{2}{|c|}{ Yes } \\
\hline Observations & \multirow{2}{*}{\multicolumn{2}{|c|}{$\begin{array}{c}572 \\
0101 / 0128\end{array}$}} & \multicolumn{2}{|c|}{572} \\
\hline Marginal $\mathrm{R}^{2}$ / Conditional $\mathrm{R}^{2}$ & & & \multicolumn{2}{|c|}{$0.082 / 0.219$} \\
\hline
\end{tabular}

Table 2: Mixed Models for Minority Influence. The VAE bot was successful at increasing minority influence when the majority cluster faced difficult decisions and majority players were thus most uncertain about their responses. The VAE bot was also successful at increasing 2-person minority cluster's influence on the mid-sized 3-person group.

Mirroring the above analysis, we also tested whether the majority was less influential in VAE teams than in control teams (the construction of majority influence variable is described in the Supporting Information) controlling for round difficulty for participants in the non-majority predictor categories. We found no main effect of treatment but once again an interaction with round difficulty. In particular, we found a reduced influence of the majority sub-group on the minority sub-group with increasing round difficulty $(\beta=-0.14, p=.03)$.

Overall, these findings show that the minority group had greater influence on the majority group in VAE teams compared to control teams. On the contrary, the majority had less influence on the minority group. These effects were modulated by round difficulty as expected by the fact that people are more prone to social influence when facing difficult decisions. These findings also suggest that the presence of a VAE bot supporting minority views more than majority made the minority opinion more resistant to social influence and more influential in difficult rounds.

\begin{tabular}{|c|c|c|c|c|}
\hline \multirow{2}{*}{$\begin{array}{l}\text { Dependent Variable: } \\
\text { Predictors }\end{array}$} & \multicolumn{2}{|c|}{ Majority Influence ${ }^{\text {minority }}$} & \multicolumn{2}{|c|}{ Majority Influence ${ }^{\text {mid-size }}$} \\
\hline & Estimate & $p$-value & Estimates & $p$-value \\
\hline (Intercept) & 0.07 & 0.457 & 0.09 & 0.457 \\
\hline Treatment & -0.13 & 0.305 & -0.23 & 0.191 \\
\hline Minority Difficulty & $0.21 * * *$ & $<0.001$ & & \\
\hline Treatment : Minority Difficulty & $-0.14 *$ & 0.036 & & \\
\hline Mid-Size Difficulty & & & $0.13 * *$ & 0.006 \\
\hline Treatment : Mid-Size Difficulty & & & 0.10 & 0.112 \\
\hline Random effects & \multicolumn{2}{|c|}{ Yes } & \multicolumn{2}{|c|}{ Yes } \\
\hline Observations & \multirow{2}{*}{\multicolumn{2}{|c|}{840}} & \multirow{2}{*}{\multicolumn{2}{|c|}{840}} \\
\hline Marginal $\mathrm{R}^{2}$ / Conditional $\mathrm{R}^{2}$ & & & & $0.047 / 0.226$ \\
\hline
\end{tabular}

Table 3: Mixed Models for Majority Influence. The VAE bot significantly reduced the majority cluster's influence on the 2-person minority cluster. A significant interaction between treatment and difficult was also found, suggesting that presence of the VAE bot further reduced majority cluster's influence in difficult trials.

Individual prediction accuracy We tested whether the presence of a minority-supporting bot affected the prediction accuracy of human participants. We ran a regression model to compare changes in prediction errors after social interaction in teams with a VAE algorithmic player versus teams with a randomizing bot. We controlled for other factors affecting prediction error like round difficulty, learning success during the training phase, and quality of social information. 
Prediction error was defined as the absolute value of the difference between predictions provided by individual $i$ in round $k$, and the round outcome indicator (Rain=1; No Rain=0):

$$
\text { prediction }_{\text {error }}^{i, k}=\mid \text { prediction }_{i, k}-\text { outcome }_{k} \mid
$$

$\Delta$ error is the change in prediction error from initial to the final forecast provided by a participant. A larger value of $\Delta$ error indicates a larger accuracy improvement. The variable is defined as:

$$
\text { improvement }_{i, k}=\Delta \text { error }_{i, k}=\text { prediction error }_{i, k}^{\text {initial }}-\text { prediction error }_{i, k}^{\text {final }}
$$

where initial and final indicate predictions made before or after seeing other team members' forecasts.

We identified three explanatory variables that could confound accuracy improvements due to our manipulation, namely (1) the accuracy of other team members' predictions in a given round (see Supplementary Information §Collective error), (2) the round's difficulty (see Supplementary Information §Round Difficulty) and (3) how well a participant had learned the relationship between predictor values and rain probability during training (see Supplementary Information $\S$ Training Error).

In addition to the above defined explanatory variables, we also control for the effect of a participant being in either the temperature, humidity, or wind predictor groups. Given that these three predictors have a different underlying relationship with rain probability, we include interaction terms between these indicator group dummy variables and the presence of a VAE bot. Including this interaction term is important, since we may expect that our treatment affects participants differently based on which predictor group they belong to. We standardized all continuous variables.

We ran a mixed regression model on $\Delta$ error (Table S6). We also report the same model run on the sign of $\Delta$ error, $\Delta$ dummy, representing binary improvement (i.e.whether accuracy improved or not) and raw final prediction error (representing final round accuracy) - see Tables S7]S9

The results show a positive trend $(\beta=0.16, p=.06$ ) for the VAE bot condition, indicating weak accuracy improvement due to the presence of the VAE bot, and a significant interaction between treatment and wind condition $(\beta=-0.26, p=$ 0.03), suggesting that the accuracy improvement was negatively affected by being assigned to a wind predictor (arguably the most difficult predictor-outcome relationship to learn). These results were also confirmed when we fitted a logistic mixed model with $\Delta$ dummy as the outcome variable. Results were robust to an alternative round difficulty measure (absolute value of $\mathrm{z}$-scores).

As expected, training error, collective error, and round difficulty all increased prediction error $(p<0.001)$. Furthermore, a higher collective error decreased the chance that a participant provided an improved forecast after seeing peer forecasts $(p<0.001)$ (Table S4).

To better understand what drove these accuracy improvements, we ran the same models on subsets of our sample based on which predictor group the participant belonged to. The results show that significant improvements due to VAE presence were driven by participants assigned to the humidity condition (Tables S5 S7). As the humidity condition was arguably the easiest to learn (positive linear), we interpret these results as suggesting that greater accuracy improvements were observed in those participants who were more likely to have learned the relationship of their indicator variable with the outcome. When dividing our sample based on which predictor group, we found that VAE significantly increased individual accuracy when the 2-person minority was assigned to the quadratic "wind" indicator variable (Tables S6 S7). In all other models, the only significant predictors of individual accuracy improvement were round difficulty, training error and collective error (Tables S5 S7). Overall, these findings suggest that gains on individual accuracy due to the VAE bot were small but consistent.

A caveat of this analysis is the high attrition rate observed during the initial forecast, which thus reduced the number of observations where a $\Delta$ error measure could be computed.

Team performance Further to the above models where we look at round-level observations from individual participants, we tested for differences in team accuracy between control and treatment teams. We defined team accuracy as the median team prediction error. When looking at aggregate data, we did not observe any significant difference in median error between treatment teams and median increase in prediction accuracy compared to control teams (both using a two-sample t-test $(p>.05)$ and a Wilcoxon test $(p>.05)$ (Figure 4).

We used two different dependent variables when testing whether our treatment had a significant effect on team performance: median team error and change in median team error. We calculated the former in a similar way as we calculated prediction error for round-level individual forecasts. We took the median prediction from a team and 
a

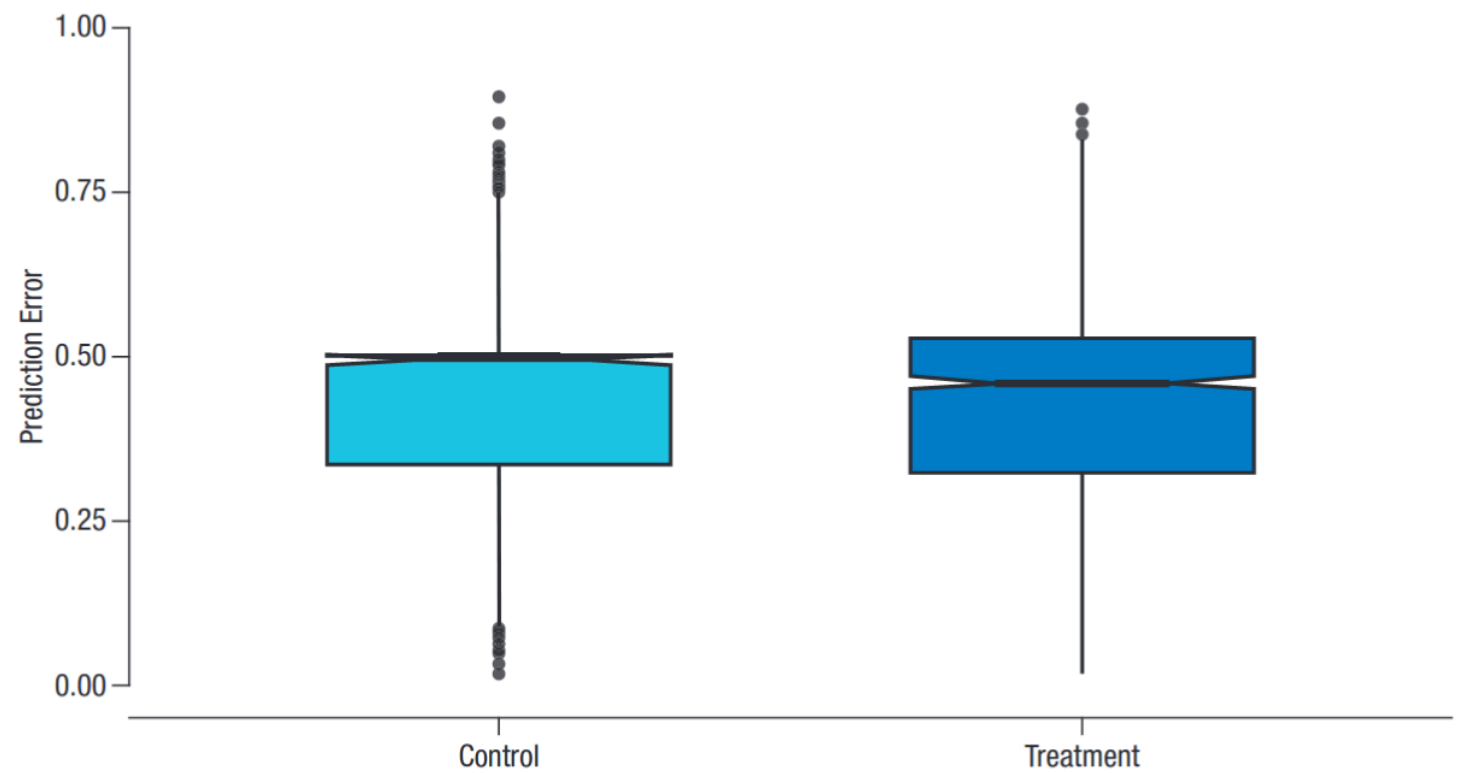

b

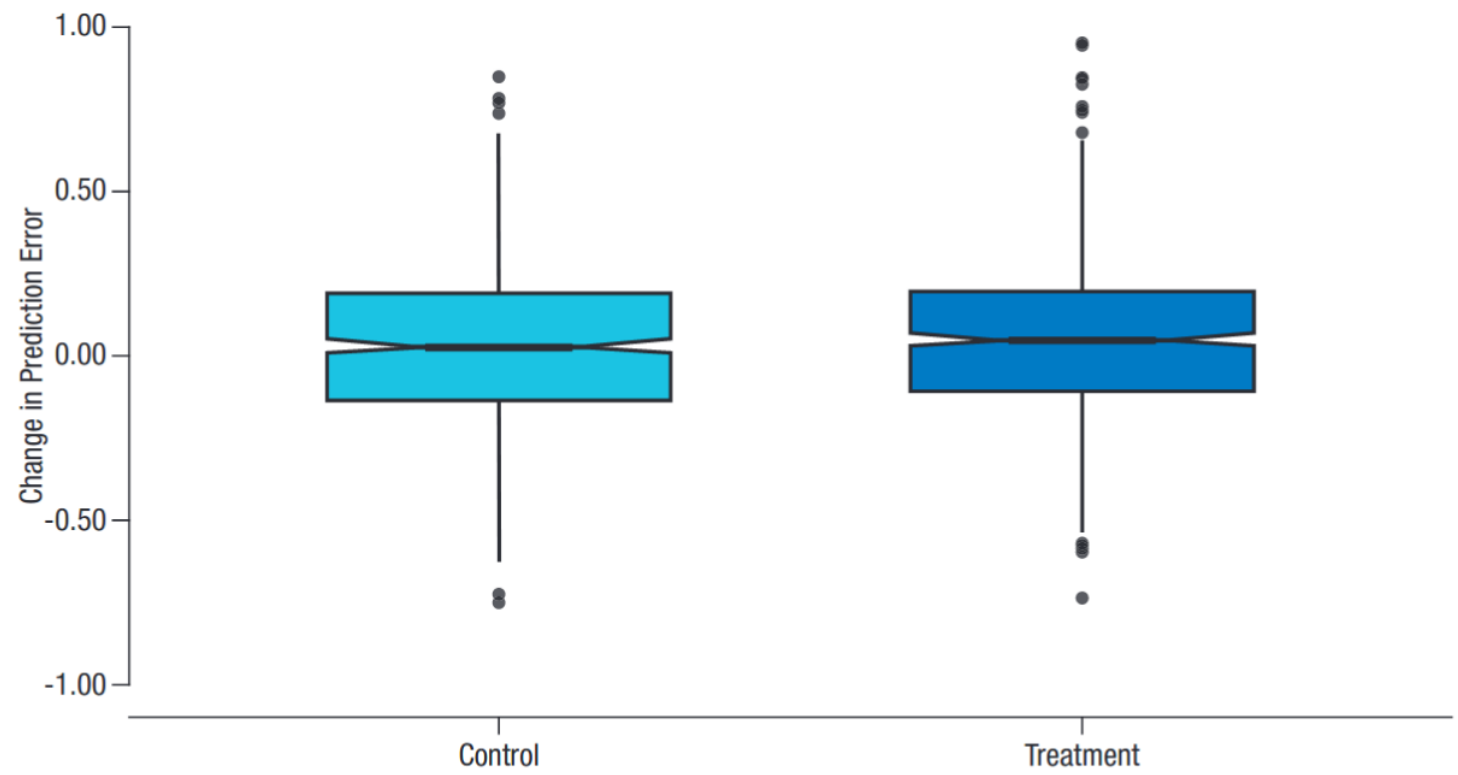

Figure 4: Boxplot of Team Median Prediction Errors (top panel) and the Difference between Initial and Final Median Prediction Error (bottom panel)

calculated the error from this prediction. For the analysis, we are only interested in the effect of bot treatment on team accuracy exclusive of bot accuracy. For comparison, however, we report results both when predictions by bots were excluded (median error) and included (median error (bot)).

$$
\operatorname{median}_{\text {error }}^{j, k}=\mid \operatorname{med}\left(\text { prediction }_{j, k}^{\text {final }}\right)-\text { outcome }_{k} \mid
$$


Our second variable of interest was $\Delta$ median error, which measured the improvement from the median initial prediction to the median final prediction. The variable was computed for team $j$ in round $k$ as follows:

$$
\Delta \text { median } \operatorname{error}_{j, k}=\mid \operatorname{med}\left(\operatorname{prediction}_{j, k}^{\text {initial }}\right)-\text { outcome }_{k}|-| \operatorname{med}\left(\operatorname{prediction}_{j, k}^{\text {final }}\right)-\text { outcome }_{k} \mid
$$

Similarly to median error, bot predictions were excluded for $\Delta$ median error and were included for $\Delta$ median error (bot).

We controlled for the same variables that were used in individual-level accuracy. Initial collective error was measured by the same variable as in the individual mixed effects models Collective Error, while round difficulty was simply the weighted average of the Round Difficulty measure used at the individual level. The weights of wind, temperature and humidity difficulty were based on the number of people in each sub-group ${ }^{2}$.

To control for training success, we summed up all the prediction errors of the last 55 training rounds in a team, and then divided the sum by 550 ( 55 rounds $\times 10$ participants per team):

$$
\text { training } \text { error }_{j}=\frac{\sum_{k=56}^{110} \sum_{i=1}^{10}{\text { prediction } \text { error }_{i, k}}_{550}}{550}
$$

Where $i \in I_{j}$ denotes participants in team $j$.

In addition to the above three control variables, we included interaction terms between the treatment variable and two further dummy variables indicating whether a group had two participants (the smallest possible group) in the wind condition or in the temperature condition. The models included a random effect for team, nested in the date the game took place. The full model is reported in Table S8.

The results presented in Table 4 show a marginal negative effect of our treatment on team medianerror $(p<.1)$ for teams when temperature was assigned to the smallest minority group $(\mathrm{N}=2)$. However, this effect was not replicated with $\Delta$ median error.

These findings suggest that wind minority groups in the random control condition got worse after social exposure. A trend was observed indicating that our treatment marginally reversed this effect, thus positively affecting accuracy improvements in this condition $(p=.06)$. Overall, these findings suggest that improvements on team accuracy were weak and constrained by what indicator variable the 2-person minority group was assigned to. However, the VAE bot

\begin{tabular}{|c|c|c|c|c|c|c|c|c|}
\hline \multirow{2}{*}{$\begin{array}{l}\text { Dependent Variable: } \\
\text { Predictors }\end{array}$} & \multicolumn{2}{|c|}{ median error } & \multicolumn{2}{|c|}{ median error (bot) } & \multicolumn{2}{|c|}{$\Delta$ median error } & \multicolumn{2}{|c|}{$\Delta$ median error (bot) } \\
\hline & Estimate & $p$-value & Estimate & $p$-value & Estimate & p-value & Estimate & $p$-value \\
\hline (Intercept) & -0.10 & 0.380 & -0.10 & 0.332 & 0.12 & 0.212 & 0.09 & 0.319 \\
\hline Treatment & 0.24 & 0.153 & 0.19 & 0.249 & -0.17 & 0.266 & -0.17 & 0.205 \\
\hline Temp. Minority & 0.18 & 0.311 & 0.17 & 0.326 & -0.26 & 0.096 & -0.13 & 0.348 \\
\hline $\begin{array}{l}\text { Treatment : Temp. Mi- } \\
\text { nority }\end{array}$ & $-0.49^{\prime}$ & 0.083 & -0.38 & 0.173 & 0.38 & 0.133 & 0.28 & 0.207 \\
\hline Wind Minority & 0.13 & 0.374 & 0.15 & 0.292 & -0.25 & 0.059 & -0.12 & 0.287 \\
\hline $\begin{array}{l}\text { Treatment : Wind Mi- } \\
\text { nority }\end{array}$ & -0.29 & 0.163 & -0.24 & 0.242 & 0.35 & 0.067 & 0.22 & 0.194 \\
\hline Random effects & \multicolumn{2}{|c|}{ Yes } & \multicolumn{2}{|c|}{ Yes } & \multicolumn{2}{|c|}{ Yes } & \multicolumn{2}{|c|}{ Yes } \\
\hline Observations & \multirow{2}{*}{\multicolumn{2}{|c|}{$0.109 / 0.150$}} & \multirow{2}{*}{\multicolumn{2}{|c|}{$0.150 / 0.189$}} & & & & \\
\hline $\begin{array}{l}\text { Marginal / Conditional } \\
\mathrm{R}^{2}\end{array}$ & & & & & \multicolumn{2}{|c|}{$0.165 / 0.193$} & \multicolumn{2}{|c|}{$0.470 / 0.495$} \\
\hline
\end{tabular}
had a stronger effect in conditions when the 2-person minority cluster was assigned to the quadratic indicator ("wind").

Table 4: Mixed Model Estimates of Treatment Effect on Team Forecasts

\footnotetext{
${ }^{2}$ Sample calculation method for a team with five participants in the wind, three in humidity and two in the temperature condition: $(5 \times$ Round Difficulty Wind $+3 \times$ Round Difficulty Hum $+2 \times$ Round Difficulty Temp $) / 10$.
} 


\section{Discussion}

In this paper, we designed a strategic algorithmic player and deployed it along human players in hybrid teams performing a hidden-profile task. The bot was built on a variational-autoencoder trained to learn a low dimensional representation of opinion clusters based on the repeated observations of people judgments. The bot was programmed to generate original predictions on the fly that supported minority views more than majority views. We manipulated the size and information of three subgroups, namely a majority (5-person condition) and two minority subgroups (3 and 2-person conditions respectively) with a access to unique task-relevant information.

We expected a bot so designed could bridge the gap between opinion clusters and improve team accuracy. Instead, we found that the presence of a single minority supporting bot (representing only $10 \%$ of the team size) increased the polarization of the team, compared to a baseline condition where a control algorithmic player provided random guesses. Our original hypothesis was based on studies in social psychology, showing that people are most influenced by opinions that are in a "latitude of acceptance", i.e. a Goldilocks region that is not too close to their privately held opinion (which would not induce any opinion shift) and not too distant from it (which would is too distant to integrate with one's information) [4, 32]. We find evidence for an increase in the polarization of the team after the introduction of the VAE bot. This suggests that rather than bridging the gap and facilitating consensus reaching, the VAE player likely had the unintended consequence of making the minority more resilient to social influence from the majority. This result echoes some early results on persuasion and peer pressure showing that the likelihood to yield to a majority believed to be wrong dramatically decrease with each additional minority-supporting individual [1, 33]. Exposure to disagreeing opinions is rarely successful at changing one's beliefs or confidence and can sometimes have the opposite effect of entrenching people in their views event further [19, 34, 35, 36, 37]. For this reason, bots may be more effective at increasing polarization than at changing opinions [38, 39].

On the other hand, the VAE bot was unsuccessful in increasing the influence of participants in the minority conditions on the majority, suggesting that participants simply became more entrenched in their views. Yet, we also find weak but consistent evidence that the bot affected collective decision patterns in subtle ways. We found weak evidence that the VAE bot helped participants improve accuracy from their initial to final forecasts. The results in Table S6 show that this was driven by observations from temperature minority groups. These results suggest that all else being equal, minority participants assigned the temperature indicator (which had a negative linear relationship with the outcome) improved their accuracy thanks to the VAE presence more than their counterpart in the control. Our findings also show some weak evidence that the VAE bot influenced team performance, although these effects did not reach significance. The VAE bot numerically reduced final forecasting errors and error change from initial to final forecast, for specific teams (for instance in teams when the minority was assigned to a negatively linear 'temperature' indicator). However, the weak effects make interpretation of accuracy results difficult. Considering that our bot treatment represented only $10 \%$ of the entire team, these results are not surprising. Future experiments should include a larger number of strategic bots and/or stronger biases towards minority opinions [1, 33].

More importantly, our VAE algorithmic agent inferred group membership without being provided any information other than the number of clusters $(k)$ to segment human players. However, contrary to simple clustering algorithms, the use of a variational auto-encoder made possible to sample new unseen responses from the hidden layer representing other players. This is in stark contrast with bots that simply copy human behavior. Variational auto-encoders reduce redundancy in the input in a similar way as a principal component analysis. Thus, the VAE model in our experiment likely inferred group membership by leveraging on the correlation of people forecasts over time. The model then used this learned internal representation to place itself along this hidden space so to support minority views proportionally more than majority views. This strategy was intended to maximally influence collective dynamics by facilitating consensus reaching. Although our algorithmic manipulation was not successful at reducing conflict, it did influence team dynamics, from increasing polarization to influencing individual and collective patterns of decision accuracy.

The growing accessibility of machine learning tools and large amount of data has significantly increased the sophistication of algorithmic agents online. Arguably, online platforms are more likely to block unsophisticated bots than bots that are better able to mimic human behavior and opinions. This trend might, over time, create a selection process favoring 'more human' sophisticated bots. Here, we explored how an adaptive algorithmic agents (using off-the-shelf machine learning techniques) could affect team dynamics and decision-making in a controlled environment. The consequences of smarter bots on collective dynamics is largely unknown. Understanding them will require conducting more experiments like the present work. Randomized treatments, both in the lab and in larger field experiments on real online platforms, can shed light on these complex dynamics. Even though our bot was explicitly designed to improve team performance in the hidden-profile task, the results were surprising. Our findings suggest that algorithms in hybrid teams interact in complex ways with the way that people perceive, share and integrate information to solve common goals. 
Notwithstanding the novelty of the results, we must stress the fact that our analysis was exploratory in nature and many caveats exist in our paradigm. First and foremost, we observed a large rate of non-compliance when participants were asked to provide initial forecasts. This may have been driven by decision fatigue or by the fact that participants in our study were not rewarded for providing accurate initial forecasts, but only accurate final forecasts. Providing performance bonus to the top two ranked participants in a team may have reduced the incentive for team-members to contribute to a shared pool of knowledge, given that this would only making other participants more likely to achieve a higher score. Decision fatigue may also be reason why we saw few initial guesses. The experimental setting required less effort to simply look at what other participants in the team guessed before providing any forecast, compared to forecasting from the initial private information as well. In short, the attentional and cognitive cost of providing initial forecasts seem to have been greater than any associated benefit.

Finally, given the bots provided initial forecasts in each round, the lack of initial guesses from human participants may have artificially increased the influence of bot forecasts on our findings. This may result in an inflated effect size compared to a scenario where all participants provide initial forecasts. Although far from perfect, we wish that our methodology can stimulate more experimental work to understand these phenomena. As seen in this work, subtle and unexpected effects may come from using off-the-shelf machine learning algorithms in hybrid social systems.

\section{Conclusions}

The increase in sophistication of social bots and the availability of off-the-shelf machine learning algorithms is likely to create a generation of smart algorithmic agents able to map opinion clusters and actively infer group membership. Generative models such as variational auto-encoders and generative adversarial networks can be used to produce bots that can strategically position themselves along arbitrary opinion spaces to maximally influence collective dynamics. In this paper, we explored how a single algorithmic agent - implemented using readily available machine learning toolscan be used to solve the hidden-profile task by strategically supporting minority views against a dominant majority.

\section{Pre-registration materials}

Pre-registered hypotheses are publicly available on the Open Science Framework: https://doi.org/10.17605/ OSF. IO/KF367

\section{Replicability}

Code and data to reproduce figures and analysis is available via the Open Science Framework: Pescetelli, N., Reichert, P., \& Rutherford, A. (2021, October 13). A variational-autoencoder approach to solve the hidden profile task in hybrid human-machine teams. Retrieved from osf.io/t38z9

\section{Acknowledgements}

We thank Alex Hustler for his support in translating the variational auto-encoder model into Keras.js and developing the web-interface.

\section{References}

[1] Solomon E. Asch. Studies of Independence and Conformity: a Minority of One Against a Unanimous Majority. Psychological Monographs: General and Applied, 70(9, whole no. 416):1-70, 1956.

[2] Andreas Flache, Michael Mäs, Thomas Feliciani, Edmund Chattoe-Brown, Guillaume Deffuant, Sylvie Huet, and Jan Lorenz. Models of Social Influence: Towards the Next Frontiers. Journal of Artificial Societies and Social Simulation, 20(4), 2017.

[3] HC. Kelman. Compliance, identification, and internalization: Three processes of attitude change. Journal of Conflict Resolution, 2(1):51-60, 1958.

[4] C.W. Sherif, M.S. Sherif, and R.E. Nebergall. Attitude and attitude change. W.B. Saunders Company, Philadelphia, 1965.

[5] Alessandro Bessi and Emilio Ferrara. Social Bots Distort the 2016 US Presidential Election Online Discussion. SSRN, 21(11), 2016. 
[6] Michiko Kakutani. The Death of Truth: Notes on Falsehood in the Age of Trump. Harper Collins, 2018.

[7] Onur Varol, Emilio Ferrara, Clayton Davis, Filippo Menczer, and Alessandro Flammini. Online Human-Bot Interactions: Detection, Estimation, and Characterization Authors. In Eleventh International AAAI Conference on Web and Social Media, page Vol. 11 No. 1, 2017.

[8] V.S. Subrahmanian, Amos Azaria, Skylar Durst, Vadim Kagan, Aram Galstyan, Kristina Lerman, Linhong Zhu, Emilio Ferrara, Alessandro Flammini, and Filippo Menczer. The DARPA Twitter Bot Challenge. Computer, 49(6):38-46, 62016.

[9] Stefan Stieglitz, Florian Brachten, Björn Ross, and Anna-Katharina Jung. Do Social Bots Dream of Electric Sheep? A Categorisation of Social Media Bot Accounts. 102017.

[10] Ken Dunham and Jim Melnick. Malicious Bots: An Inside Look into the Cyber-Criminal Underground of the Internet. CRC Press, Boca Raton, 2009.

[11] Kristina Lerman, Xiaoran Yan, and Xin-Zeng Wu. The "Majority Illusion" in Social Networks. PLOS ONE, 11(2):e0147617, 22016.

[12] Malte F. Jung, Nikolas Martelaro, and Pamela J. Hinds. Using Robots to Moderate Team Conflict. In Proceedings of the Tenth Annual ACM/IEEE International Conference on Human-Robot Interaction, New York, NY, USA, 3 2015. ACM.

[13] Levin Brinkmann, Deniz Gezerli, Kira von Kleist, Thomas F. Müller, Iyad Rahwan, and Pescetellim Niccolò. Human biases limit algorithmic boosts of cultural evolution. Philosophical Transactions of the Royal Society A, 2021.

[14] Garold Stasser and William Titus. Hidden Profiles : A Brief History. Psychological Inquiry, 14(3):304-313, 2003.

[15] John P. Lightle, John H. Kagel, and Hal R. Arkes. Information Exchange in Group Decision Making: The Hidden Profile Problem Reconsidered. Management Science, 55(4):568-581, 42009.

[16] Massimo Stella, Emilio Ferrara, and Manlio De Domenico. Bots increase exposure to negative and inflammatory content in online social systems. Proceedings of the National Academy of Sciences of the United States of America, 115(49):12435-12440, 122018.

[17] Jacob Ratkiewicz, Michael Conover, Mark Meiss, Bruno Goncalves, Alessandro Flammini, and Filippo Menczer. Detecting and Tracking Political Abuse in Social Media. In Proceedings of the International AAAI Conference on Web and Social Media, page 5(1), 2011.

[18] Stefano Cresci, Roberto Di Pietro, Marinella Petrocchi, Angelo Spognardi, and Maurizio Tesconi. The ParadigmShift of Social Spambots. In Proceedings of the 26th International Conference on World Wide Web Companion WWW'17 Companion, pages 963-972, New York, New York, USA, 2017. ACM Press.

[19] Christopher A. Bail, Lisa P. Argyle, Taylor W. Brown, John P. Bumpus, Haohan Chen, M. B. Fallin Hunzaker, Jaemin Lee, Marcus Mann, Friedolin Merhout, and Alexander Volfovsky. Exposure to opposing views on social media can increase political polarization. Proceedings of the National Academy of Sciences, 115(37):9216-9221, 92018.

[20] Christopher A. Bail, Brian Guay, Emily Maloney, Aidan Combs, D. Sunshine Hillygus, Friedolin Merhout, Deen Freelon, and Alexander Volfovsky. Assessing the Russian Internet Research Agency's impact on the political attitudes and behaviors of American Twitter users in late 2017. Proceedings of the National Academy of Sciences, 117(1):243-250, 12020.

[21] Jennifer Allen, Baird Howland, Markus Mobius, David Rothschild, and Duncan J. Watts. Evaluating the fake news problem at the scale of the information ecosystem. Science Advances, 6(14):eaay3539, 42020.

[22] Andrew Guess, Jonathan Nagler, and Joshua Tucker. Less than you think: Prevalence and predictors of fake news dissemination on Facebook. Science Advances, 5(1):eaau4586, 12019.

[23] Philip Howard. How Political Campaigns Weaponize Social Media Bots. IEEE Spectrum, Oct, 2018.

[24] Emilio Ferrara, Onur Varol, Clayton Davis, Filippo Menczer, and Alessandro Flammini. The rise of social bots. Communications of the ACM, 59(7):96-104, 62016.

[25] Heidi Ledford. Social scientists battle bots to glean insights from online chatter. Nature, 578(7793):17-17, 2 2020.

[26] Irina Higgins, Le Chang, Victoria Langston, Demis Hassabis, Christopher Summerfield, Doris Tsao, and Matthew Botvinick. Unsupervised deep learning identifies semantic disentanglement in single inferotemporal face patch neurons. Nature Communications, 12(1):6456, 122021. 
[27] Mariam Orabi, Djedjiga Mouheb, Zaher Al Aghbari, and Ibrahim Kamel. Detection of Bots in Social Media: A Systematic Review. Information Processing \& Management, 57(4):102250, 72020.

[28] Hirokazu Shirado and Nicholas A. Christakis. Locally noisy autonomous agents improve global human coordination in network experiments. Nature, 545(7654):370-374, 52017.

[29] Abdullah Almaatouq, Joshua Becker, James P. Houghton, Nicolas Paton, Duncan J. Watts, and Mark E. Whiting. Empirica: a virtual lab for high-throughput macro-level experiments. Behavior Research Methods, 53(5):21582171, 102021.

[30] Jan Lorenz, Heiko Rauhut, Frank Schweitzer, and Dirk Helbing. How social influence can undermine the wisdom of crowd effect. Proceedings of the National Academy of Sciences of the United States of America, 108(22):9020-5, 52011.

[31] Aaron Bramson, Patrick Grim, Daniel J. Singer, Steven Fisher, William Berger, Graham Sack, and Carissa Flocken. Disambiguation of social polarization concepts and measures. The Journal of Mathematical Sociology, 40(2):80-111, 42016.

[32] Ilan Yaniv and Maxim Milyavsky. Using advice from multiple sources to revise and improve judgments. Organizational Behavior and Human Decision ..., 103:104-120, 2007.

[33] Bibb Latane. The psychology of social impact. American Psychologist, 36(4):343-356, 1981.

[34] Niccolò Pescetelli and Nick Yeung. The effects of recursive communication dynamics on belief updating. Proceedings of the Royal Society B: Biological Sciences, 287(1931):20200025, 72020.

[35] John Zaller. The Nature and Origins of Mass Opinion. Cambridge University Press, Cambridge, UK, 1992.

[36] Kyle Endres and Costas Panagopoulos. Cross-Pressure and Voting Behavior: Evidence from Randomized Experiments. The Journal of Politics, 81(3):1090-1095, 72019.

[37] Joshua L. Kalla and David E. Broockman. The Minimal Persuasive Effects of Campaign Contact in General Elections: Evidence from 49 Field Experiments. American Political Science Review, 112(1):148-166, 22018.

[38] Leo G. Stewart, Ahmer Arif, and Kate Starbird. Examining Trolls and Polarization with a Retweet Network. In Proc. ACM WSDM, Workshop on Misinformation and Misbehavior Mining on the Web, 2018.

[39] David A. Broniatowski, Amelia M. Jamison, SiHua Qi, Lulwah AlKulaib, Tao Chen, Adrian Benton, Sandra C. Quinn, and Mark Dredze. Weaponized Health Communication: Twitter Bots and Russian Trolls Amplify the Vaccine Debate. American Journal of Public Health, 108(10):1378-1384, 102018. 
489

\section{Supplementary Figures}
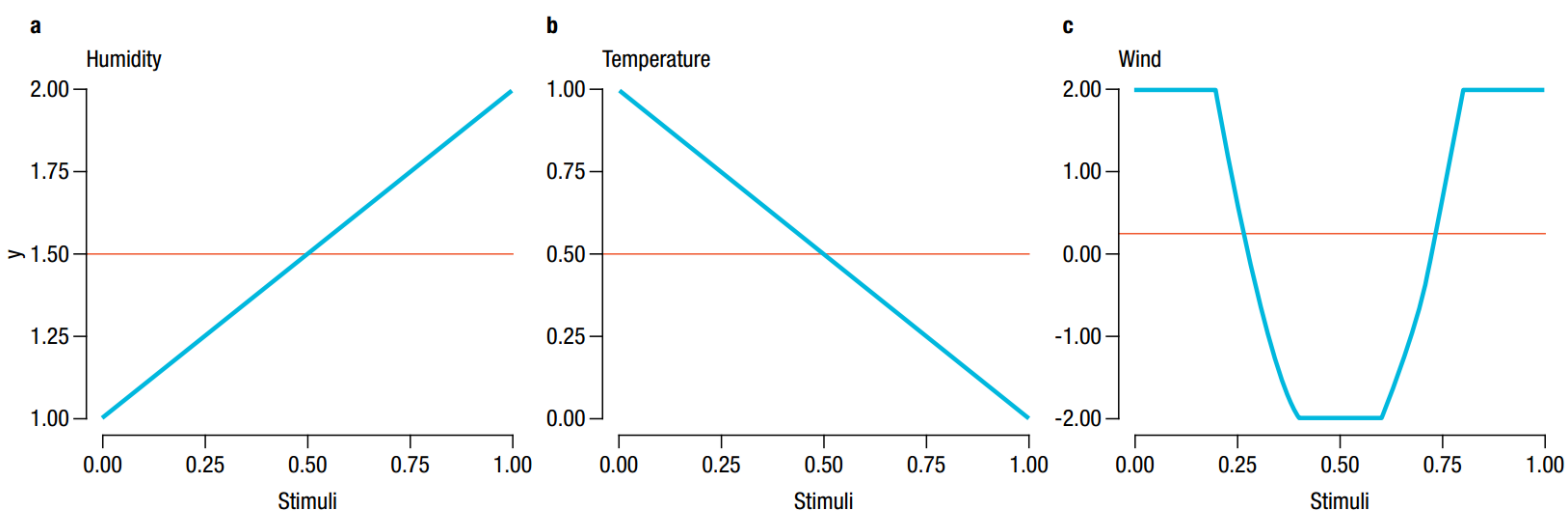

Figure S1: Predictor functions on the $[0,1]$ interval (pink horizontal line represents the function average on this interval) 
A PREPRINT - November 18, 2021

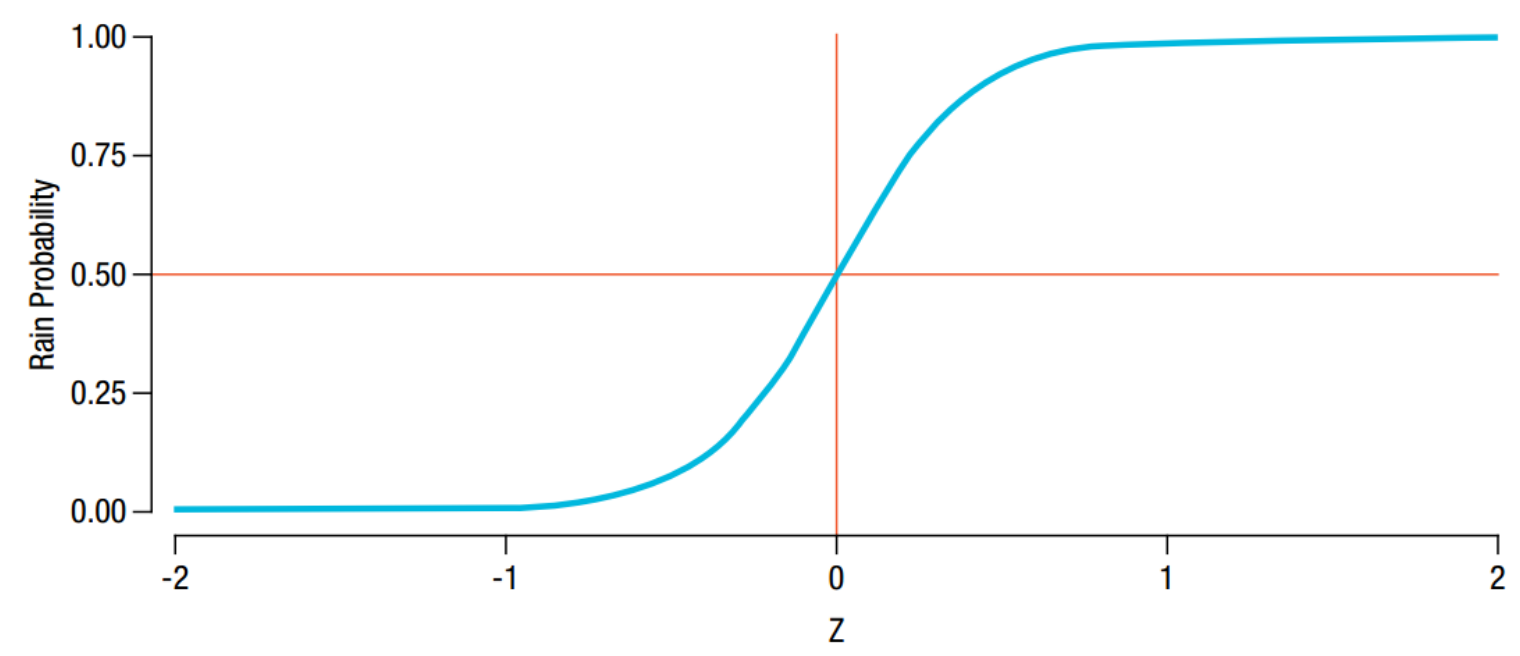

Figure S2: Sigmoid Function Calculating Rain Probability 


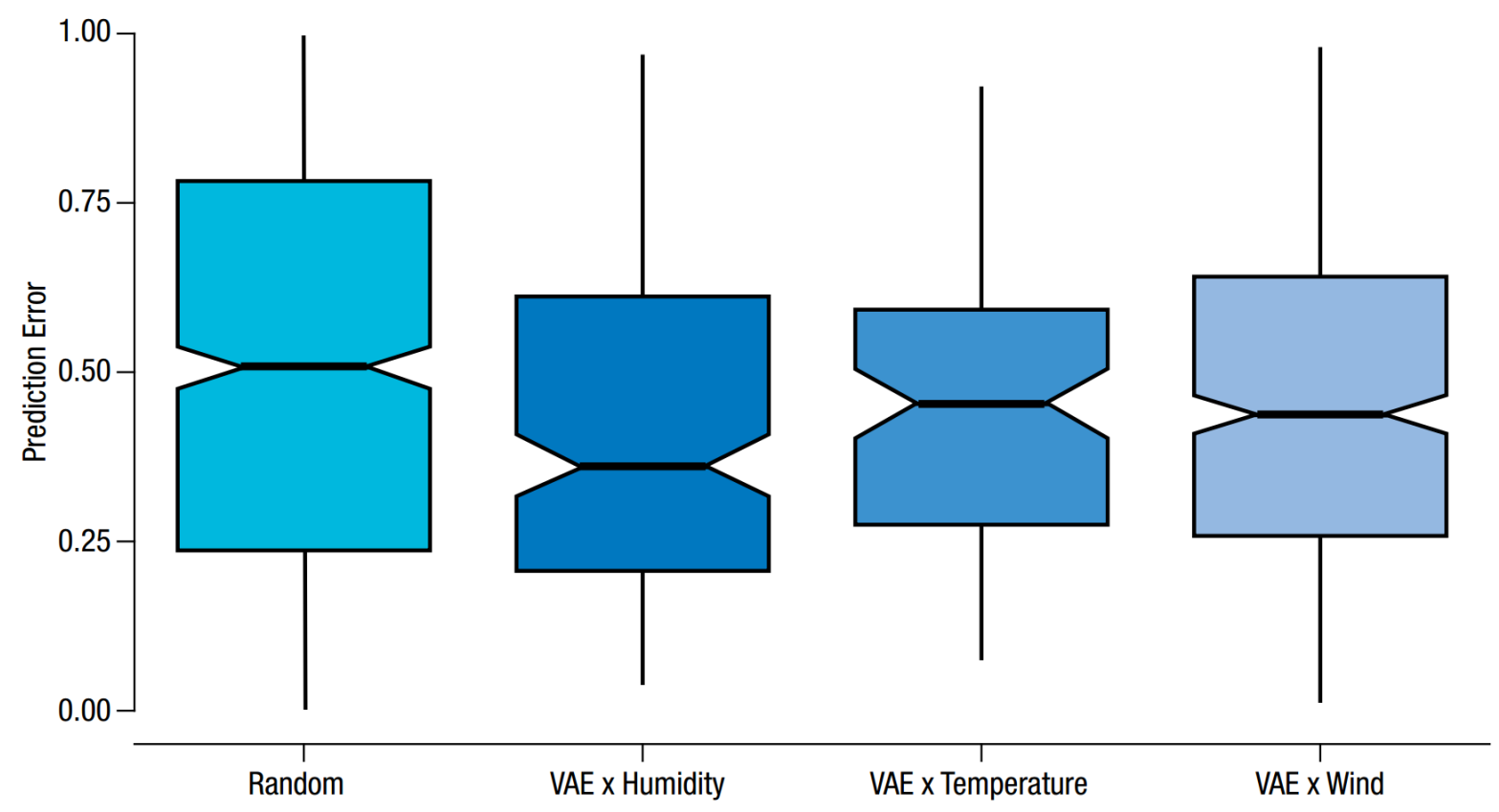

Figure S3: Prediction Error by Algorithmic players: Random bots vs. VAE (separated by which predictor group was in the minority) 


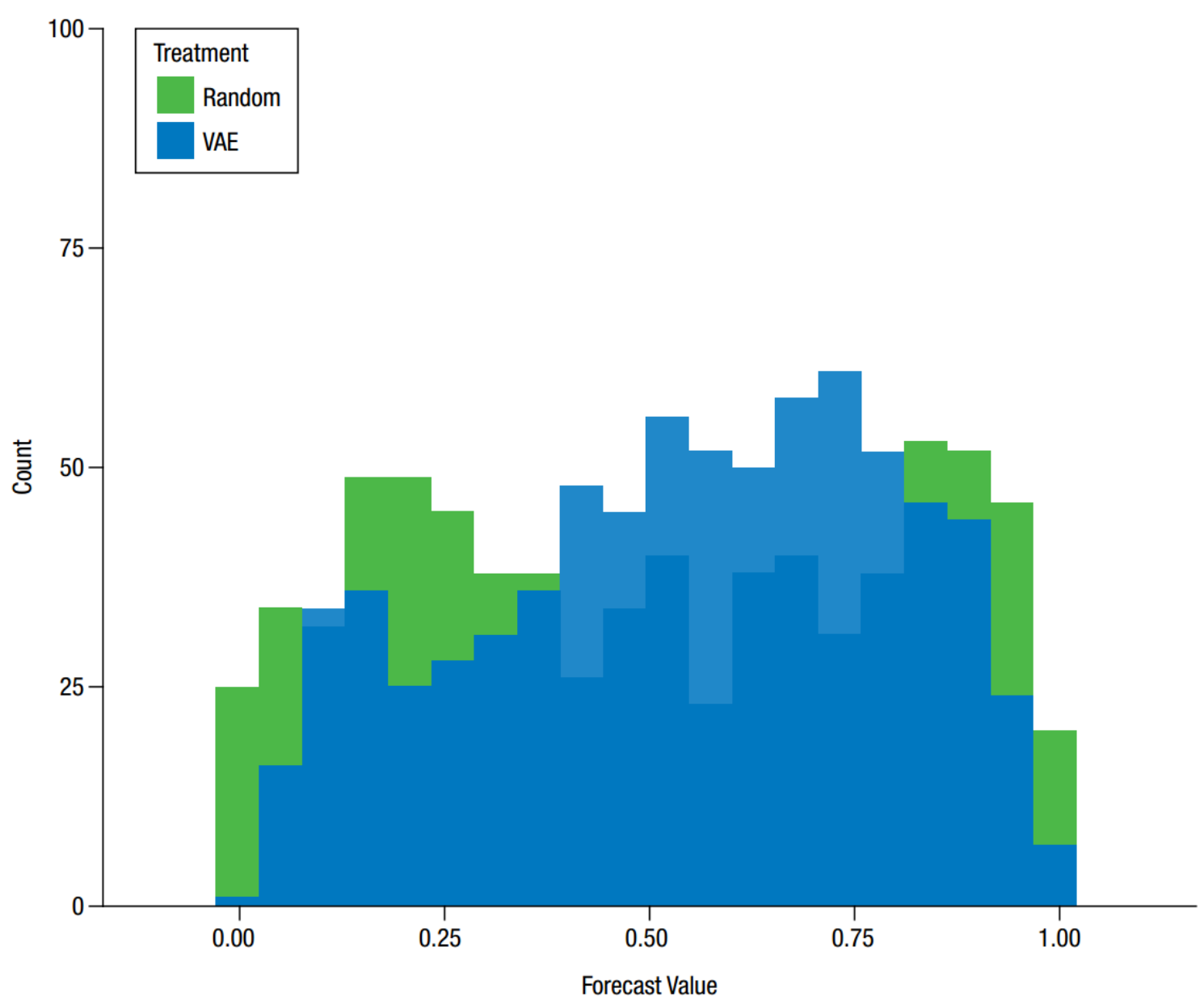

Figure S4: Histogram of Predictions: Random vs. VAE 


\section{VAE Bot versus Random Forecasts}

As a start of our analysis, we want to understand how the VAE model performs versus randomly generated guesses. Figure S3 shows that VAE bots provide lower prediction error than randomized answers, with the $95 \%$ confidence intervals of the median not overlapping for bots trained in humidity and wind groups. The boxplots show that VAE with humidity predictor group as the minority performs the best, while wind and temperature perform approximately the same.

To test formally if the trained model has better accuracy compared to random predictions, we run a simple OLS estimation where we regress the treatment dummy ("VAE", 1 if prediction was provided by the VAE algorithm, 0 if it was provided by the randomizing bot), average round difficulty, and dummies for wind and humidity minority on prediction error. The significant $(\mathrm{p}<0.001)$ negative coefficient on the treatment dummy indicates that the VAE model provides forecasts with lower prediction error (see Table $\mathrm{S} 2$ for results), representing an improvement of $11.5 \%$.

We can observe that the trained model's predictions are closer to a bell-shaped distribution with a positive skew, while the randomized predictions are more skewed towards the extremes (see Figure S4).

\section{Round Difficulty}

The predictor functions include a random noise term, which we ignore for the below calculations. As the z-score of predictor values determines if it has a negative (negative z-score) or positive (positive z-score) effect on rain probability, we just need to find the predictor value that corresponds to the predictor function's average on the $[0,1]$ interval. Humidity and temperature have a linear positive, and linear negative relationship with rain probability, respectively. The linear relationship makes it straight-forward to calculate which predictor value provides the least amount of information to participants. The average of an either decreasing or increasing linear function on the 0,1 interval is at $x=0.5$, which is our most ambiguous stimuli value for both humidity and temperature predictors. As wind has a quadratic relationship with rain probability, we will have not one, but two "most ambiguous" values, denoted by $x_{1}$ and $x_{2}$.

The wind predictor function is given by $f(x)=50 x 2-50 x+10+\epsilon$, where $x \in[0,1]$ and $\epsilon \sim N(0,1)$, but $f(x)=2$ if $x \leq 2$ and $0.8 \leq x \leq 1$, furthermore, $f(x)=-2$ if $x \leq-2$. The alternative representation of this function (setting aside the random noise term) is:

$$
f(x)= \begin{cases}2, & 0 \leq x<0.2 \\ 50 x^{2}-50 x+10, & 0.2 \leq x<0.4 \\ -2, & 0.4 \leq x<0.6 \\ 50 x^{2}-50 x+10, & 0.6 \leq x<0.8 \\ 2, & 0.8 \leq x \leq 1\end{cases}
$$

When calculating the function average for $f(x)$ on interval $[\mathrm{a}, \mathrm{b}]$, we use the following well-known formula:

$$
\bar{f}_{a, b}=\frac{1}{b-a} \int_{a}^{b} f(x) d x
$$

Following the above calculations, we obtain $x_{1}=0.264755$ and $x_{2}=0.735245$. After we calculated the most ambiguous stimuli for all three predictor categories, we then measure round difficulty by the relative distance of the stimuli from the most ambiguous stimulus or stimuli (note that the calculation of wind difficulty depends on which interval the wind stimuli belongs to):

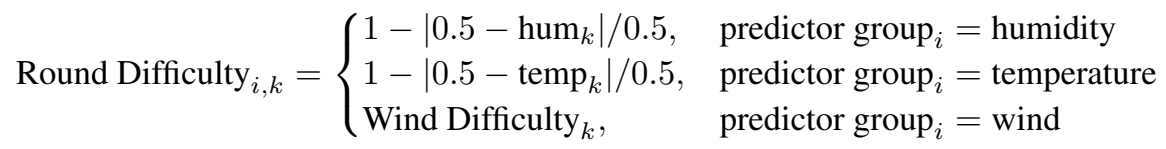


Where Wind Difficulty is calculated as:

$$
\text { Wind Difficulty }_{k}= \begin{cases}1-\left[\left(x_{1}-\operatorname{wind}_{k}\right) / x_{1}\right], & \operatorname{wind}_{k}<x_{1} \\ 1-\left[\left(0.5-\operatorname{wind}_{k}\right) /(0.5-x 1)\right], & x_{1} \leq \operatorname{wind}_{k}<0.5 \\ 1-\left[\left(x_{2}-\operatorname{wind}_{k}\right) /\left(x_{2}-0.5\right)\right], & 0.5 \leq \operatorname{wind}_{k}<x_{2} \\ 1-\left[\left(\operatorname{wind}_{k}-x_{2}\right) /\left(1-x_{2}\right),\right. & x 2 \leq \operatorname{wind}_{k}\end{cases}
$$

Essentially, we measure the percentage deviation from the most "ambiguous points" of each predictor value (humidity, temperature, wind) in a round. By subtracting the resulting value from 1, we have a higher value of Round Difficulty ${ }_{i, k}$ indicating a more difficult round for participant $i$.

\section{Majority Influence}

Our approach to constructing majority influence is very similar to what we used to calculate minority influence.

We calculated the absolute difference between the median of the initial majority forecasts and the median final forecast for the other two sub-group sizes (2-person minority or 3-person mid-size sub-group):

$$
\begin{aligned}
& \text { Majority Influence }_{j, k}^{\text {majority }}=1-\left|\operatorname{med}\left(\operatorname{prediction}_{I_{j, k}^{\text {mijority }}}^{\text {initial }}\right)-\operatorname{med}\left(\operatorname{prediction}_{I_{j, k}^{\text {minority }}}^{\text {final }}\right)\right| \\
& \text { Majority Influence }_{j, k}^{\text {mid-size }}=1-\left|\operatorname{med}\left(\operatorname{prediction}_{I_{j, k}^{\text {mijarity }}}^{\text {initial }}\right)-\operatorname{med}\left(\operatorname{prediction}_{I_{j, k}^{\text {mind size }}}^{\text {final }}\right)\right|
\end{aligned}
$$

\section{Collective Error}

Collective error was defined as the prediction error measured from the team median prediction excluding the participant's own prediction. The collective error for participant $i$ of team $j$ in round $k$ is defined as:

$$
\text { Collective } \text { Error }_{i, j, k}=\mid \operatorname{med}\left(\text { prediction }_{I_{j, k}^{-i}, k}^{\text {initial }}\right)-\text { outcome }_{k} \mid
$$

Where the $I_{j}^{-i}$ subscript indicates the participants in team $j$ excluding participant $i$.

\section{Training Error}

In the accuracy regression models in the main text, we control for how well a participant learned the relationship between their assigned predictor and the outcome. We were interested in the participant's final learning at the end of the training phase. Therefore, we constructed training error as the average prediction error in the last half of the training phase, which corresponds to 55 round $3^{3}$

$$
\text { training } \text { error }_{i}=\frac{\sum_{k=56}^{110}{\text { prediction } \text { error }_{i, k}}_{55}}{55}
$$

\footnotetext{
${ }^{3}$ We ignore rounds where no forecast was provided by a participant (we remove these observations from both the numerator and the denominator in the equation).
} 


\section{Supplementary tables}

Table S1: Summary Statistics of Sample Demographics

\begin{tabular}{|c|c|c|c|c|c|c|}
\hline & $\mathrm{N}$ & Mean & SD & Median & Min & $\operatorname{Max}$ \\
\hline Age & 295 & 31.837 & 10.083 & 30 & 18 & 66 \\
\hline Gender & 292 & 0.524 & 0.500 & 1 & 0 & 1 \\
\hline n. of Submissions & 299 & 178.930 & 189.366 & 114 & 1 & 1149 \\
\hline Approval Ratio & 299 & 0.990 & 0.033 & 1 & 0.6 & 1 \\
\hline First Language & Percentage & Nationality & Percentage & & & \\
\hline English & 64.5 & United Kingdom & 38.8 & & & \\
\hline Other & 22.1 & Other 28.1 & & & & \\
\hline Portuguese & 7.0 & United States & 21.1 & & & \\
\hline \multirow[t]{2}{*}{ Polish } & 6.4 & Poland & 6.4 & & & \\
\hline & & Portugal & 5.7 & & & \\
\hline Employment Status & Percentage & Student Status & Percentage & & & \\
\hline Full-Time & 47.8 & No & 66.6 & & & \\
\hline Other & 23.4 & Yes & 30.8 & & & \\
\hline Part-Time & 16.4 & Other & 2.7 & & & \\
\hline Unemployed (and job seeking) & 12.4 & & & & & \\
\hline
\end{tabular}


A PREPRINT - NOVEMBER 18, 2021

Table S2: Prediction Error by Algorithmic Players: Random vs. VAE (OLS regression)

\begin{tabular}{|c|c|c|c|}
\hline \multirow[b]{2}{*}{ Predictors } & \multicolumn{3}{|c|}{ Prediction Error } \\
\hline & Estimates & $\mathrm{CI}$ & $p$-value \\
\hline (Intercept) & $0.52 * * *$ & $0.47-0.58$ & $<0.001$ \\
\hline VAE & $-0.06 * * *$ & $-0.08--0.03$ & $<0.001$ \\
\hline Avg. Difficulty & 0.03 & $-0.05-0.11$ & 0.495 \\
\hline Wind Minority & -0.03 & $-0.07-0.01$ & 0.138 \\
\hline Humidity Minority & $-0.06 * *$ & $-0.11--0.02$ & 0.003 \\
\hline Observations & 1500 & & \\
\hline$R^{2} / R^{2}$ adjusted & $0.016 / 0.014$ & & \\
\hline
\end{tabular}


Table S3: Summary of Mixed Model Estimates of Treatment Effect on full sample (full results including all predictors are reported in Tables $\mathbf{S} 4 \mathrm{~S} 7 \mathrm{P}$

\begin{tabular}{lcccccc}
\hline Dependent Variable: & \multicolumn{3}{c}{ prediction error } & \multicolumn{2}{c}{$\Delta$ error } & \multicolumn{2}{c}{$\Delta$ dummy } \\
\cline { 2 - 7 } Predictors & Estimate & p-value & Estimate & $p$-value & Odds Ratio & $p$-value \\
(Intercept) & 0.02 & 0.546 & -0.09 & 0.129 & 0.98 & 0.866 \\
Treatment & 0.00 & 0.982 & 0.16 & 0.068 & $1.41^{\prime}$ & 0.081 \\
Temperature & -0.00 & 0.977 & 0.11 & 0.206 & $1.48^{*}$ & $\mathbf{0 . 0 3 4}$ \\
Treatment : Temperature & 0.00 & 0.95 & -0.14 & 0.235 & 0.78 & 0.332 \\
Wind & -0.06 & 0.142 & 0.15 & 0.08 & $1.52^{*}$ & $\mathbf{0 . 0 2 6}$ \\
Treatment : Wind & 0.00 & 0.928 & $-0.26^{*}$ & $\mathbf{0 . 0 3 3}$ & 0.64 & 0.094 \\
\hline Random effects & \multicolumn{2}{c}{ Yes } & \multicolumn{2}{c}{ Yes } & \multicolumn{2}{c}{ Yes } \\
Logistic Mixed Model & \multicolumn{2}{c}{ No } & \multicolumn{2}{c}{ No } & \multicolumn{2}{c}{ Yes } \\
Observations & \multicolumn{2}{c}{14522} & 2536 & $0.045 / 0.179$ \\
Marginal / Conditional $\mathrm{R}^{2}$ & $0.039 / 0.172$ & $0.027 / 0.148$ & $0.045 / 0$ \\
\hline
\end{tabular}




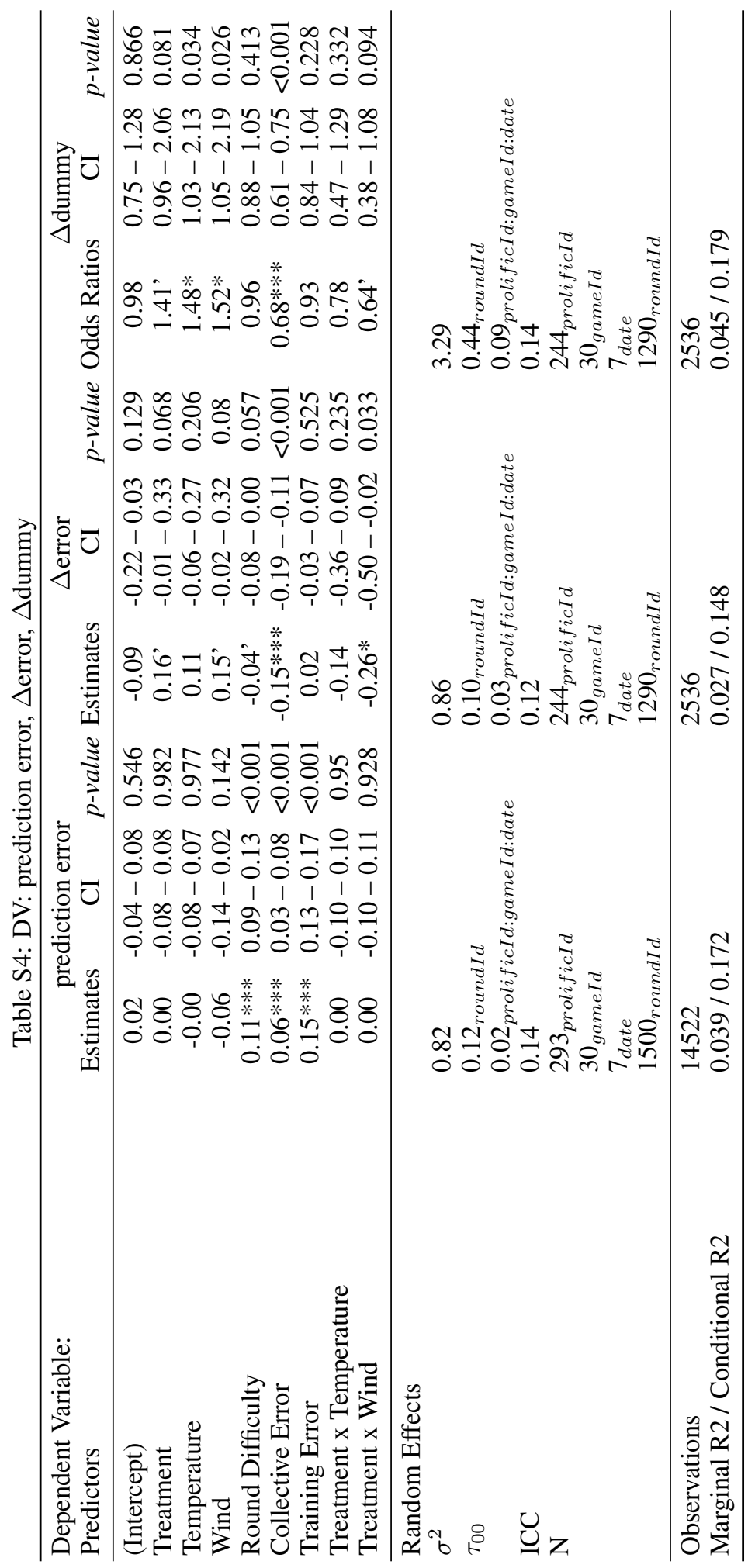




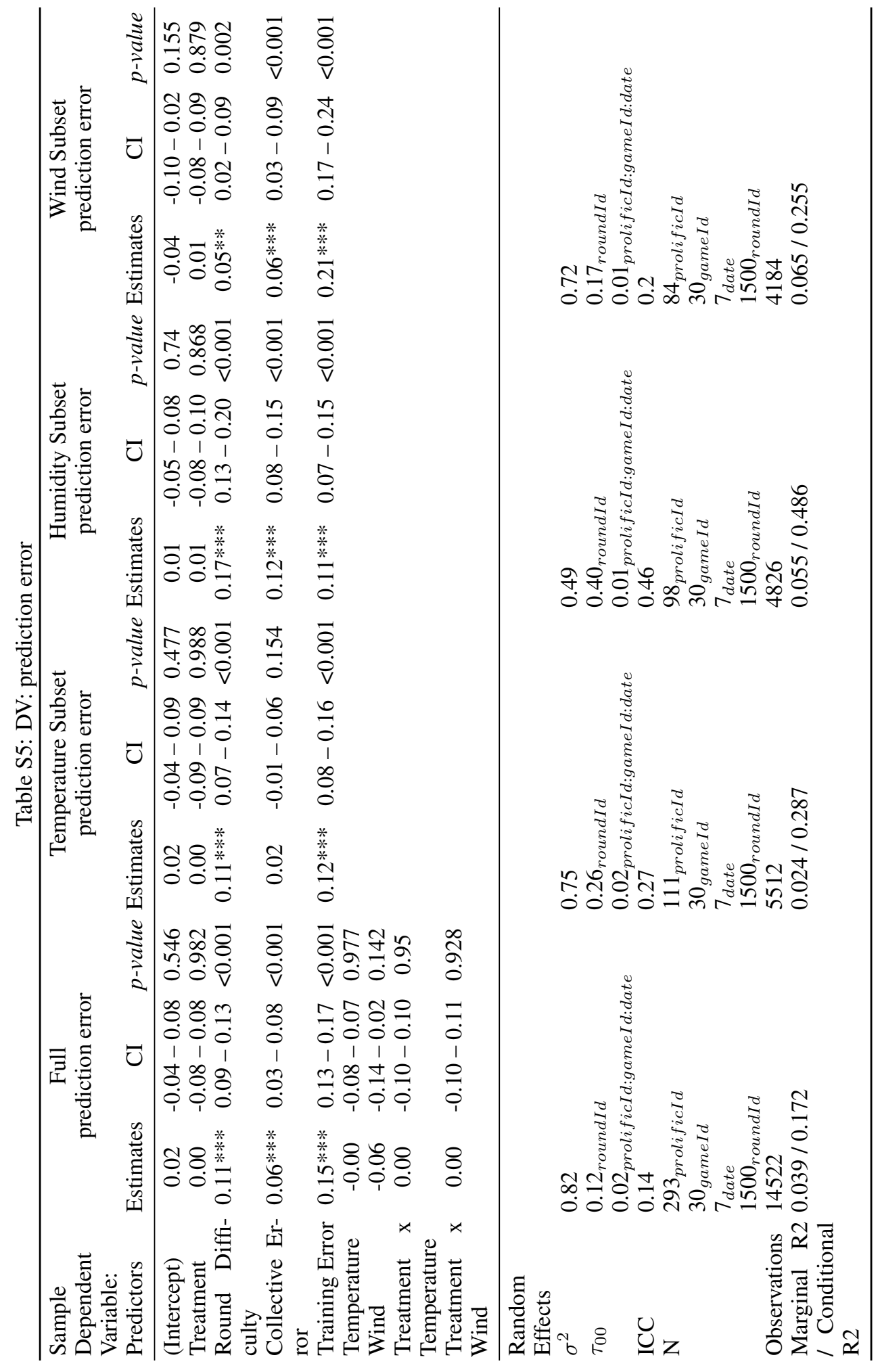




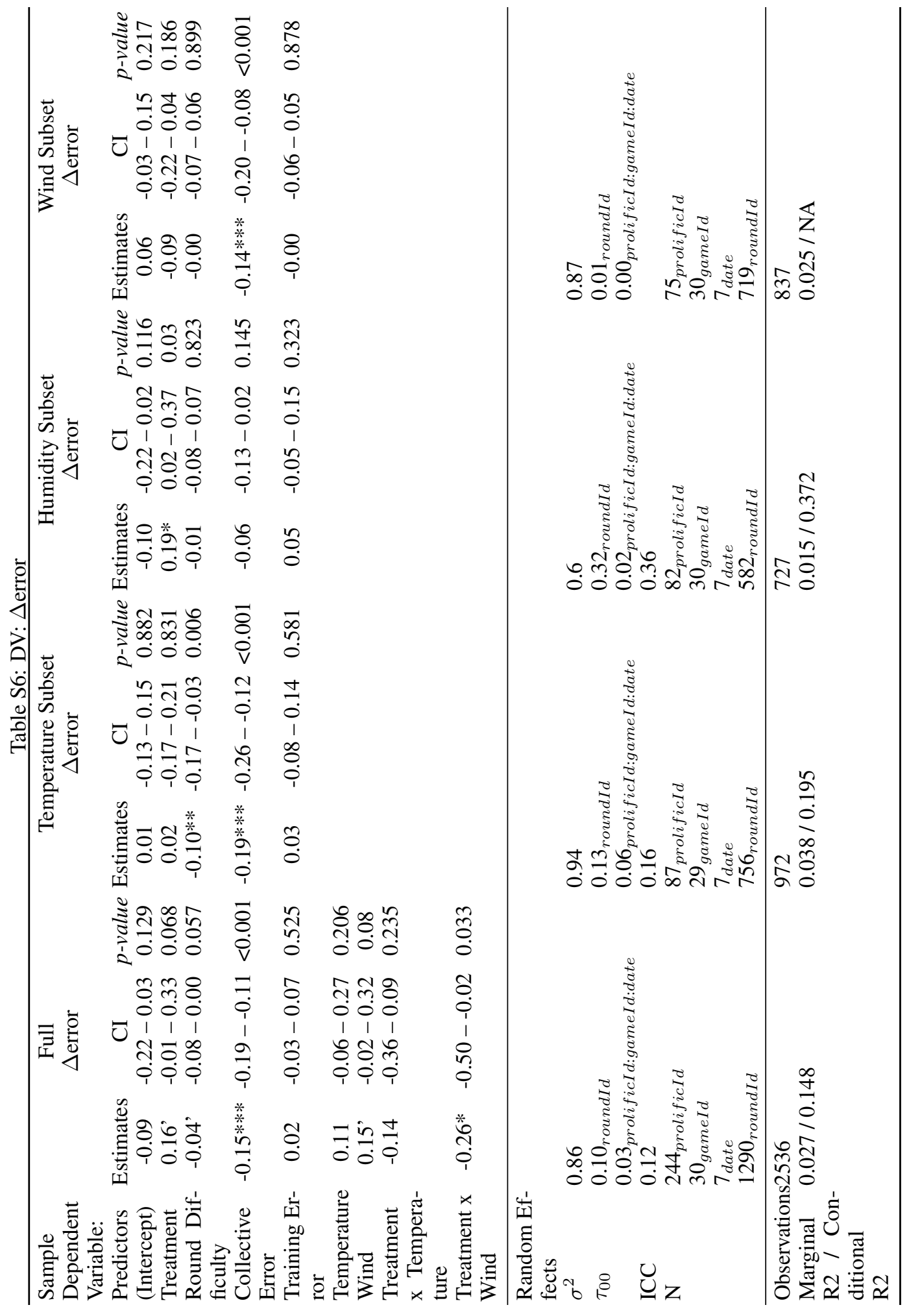




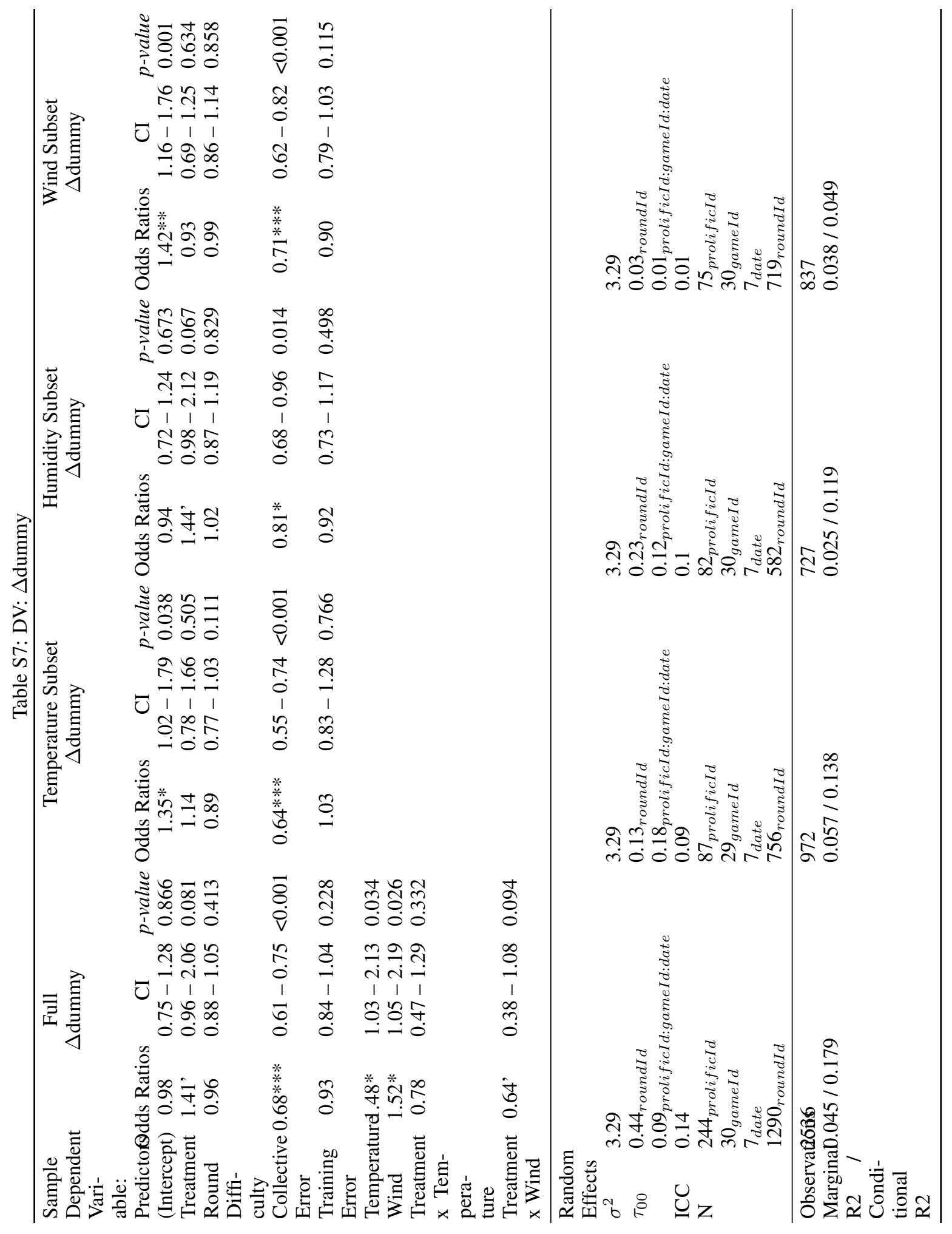




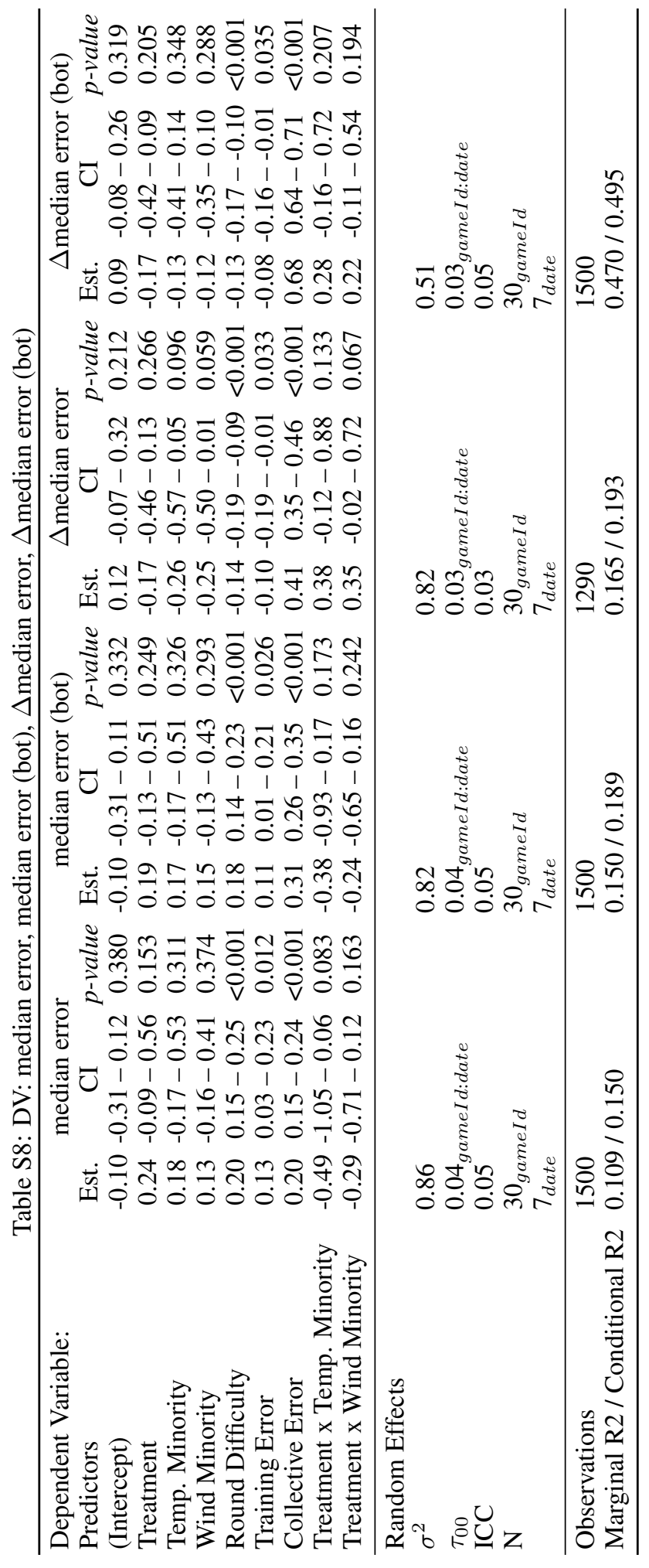




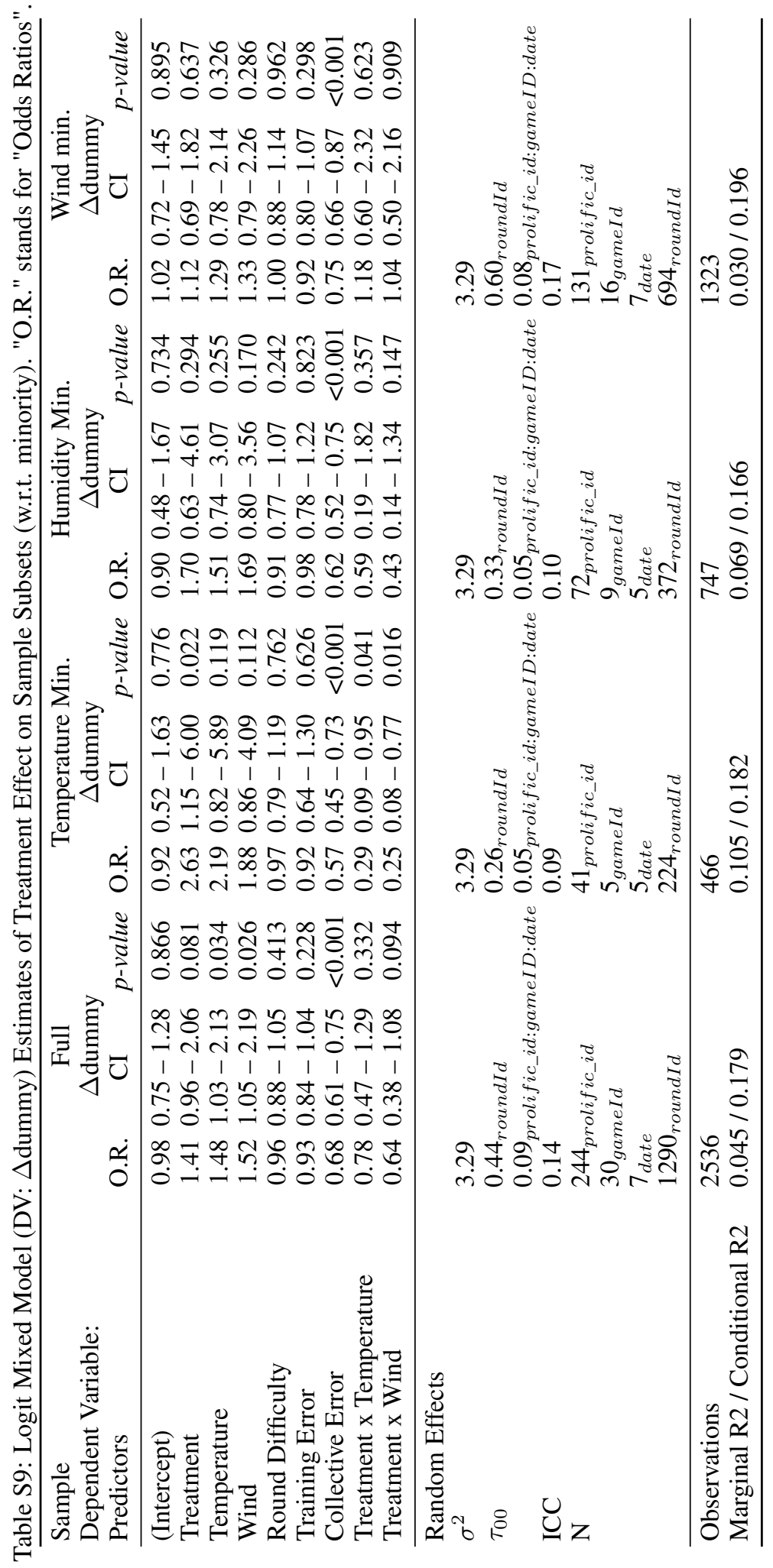

